\title{
Systematic approximation of multi-scale Feynman integrals
}

\section{Sophia Borowka, ${ }^{a}$ Thomas Gehrmann $^{b}$ and Daniel Hulme ${ }^{b}$}

a Theoretical Physics Department, CERN, CH-1211 Geneva 23, Switzerland

${ }^{b}$ Physik-Institut, Universität Zürich, Winterthurerstr. 190, CH-8057 Zürich, Switzerland

E-mail: sophia.borowka@cern.ch, thomas.gehrmann@uzh.ch, dhulme@physik.uzh.ch

ABSTRACT: An algorithm for the systematic analytical approximation of multi-scale Feynman integrals is presented. The algorithm produces algebraic expressions as functions of the kinematical parameters and mass scales appearing in the Feynman integrals, allowing for fast numerical evaluation. The results are valid in all kinematical regions, both above and below thresholds, up to in principle arbitrary orders in the dimensional regulator. The scope of the algorithm is demonstrated by presenting results for selected two-loop threepoint and four-point integrals with an internal mass scale that appear in the two-loop amplitudes for Higgs + jet production.

KEYwords: Perturbative QCD, Scattering Amplitudes

ArXIV EPRINT: 1804.06824 


\section{Contents}

1 Introduction 1

2 The algorithm 2

3 Discourse on the method $\quad 8$

4 Application to three-scale two-loop four-point integrals $\quad 19$

$\begin{array}{llr}5 & \text { Conclusion } & 29\end{array}$

\section{Introduction}

Feynman integrals [1] are a fundamental constituent of perturbative calculations in theoretical particle physics and many techniques have been developed to calculate them. Going beyond one loop, the calculation of multi-scale multi-loop integrals is still a limiting factor in the theoretical predictions of hard processes.

The use of differential equations [2-9] and expression of the resulting integrals in terms of generalised polylogarithms [10-19] has proven most successful over the past years and has led to a plethora of analytically available results [20-32], leading in turn to important phenomenological predictions. With the presence of internal massive lines or particular non-planar graphs, elliptic structures appear which cannot be expressed in terms of polylogarithms alone. While progress is being made towards a description of these [33-39], the numerical approach using sector decomposition [40-43] in publicly available programs [44-52] has become more and more viable with successful phenomenological applications to up to two-loop four-point four-scale processes [53-57]. Still, the numerical evaluation suffers from long evaluation times or it is limited in accuracy, and more often than not, a tuning of the integration parameters is needed to allow for a fastly-converging accurate result.

To shorten the evaluation times the results have to be algebraic in the kinematical parameters (Mandelstam invariants, external and internal particle masses). Then the evaluation at each kinematic point takes just as long as the time needed for the insertion of their numerical values. Algebraic results can be obtained if the integrands are Taylor expanded in the Feynman parameters. To ensure that the approximation is fastly-converging, each integrand must be manipulated so that it is in a form optimised for a Taylor expansion.

To obtain such results in an algorithmic way, and thus find a compromise between analytic and numerical approaches, TAYINT, an algorithm to analytically approximate loop integrals, and generate an algebraic integral library which can be instantaneously evaluated, is presented in this paper. The generation of the integral library is a lengthy undertaking, and the backbone of the method to do so is as follows: 
1. Input an integral.

2. Reduce the integral to a quasi-finite basis introduced in refs. $[58,59]$, such that the divergences are in the coefficient of the simplest integrals. An automated script using the libraries of the publicly available program REDUZE [59-61] performs this.

3. For the quasi-finite basis integrals, carry out a decomposition into subsectors with smoother integrands. These are obtained using the publicly available program SECDEC 3 [49-52], without its contour deformation option. The subsector integrands are analytic within the integration region, but may contain integrable singularities over thresholds and at upper integration boundaries.

4. Use a conformal mapping to move the singularities outside of the region of integration as far away as is possible. This is done in Mathematica [62]. The structure of conformal mappings is such that the singular behaviour is moved as far as possible.

5. (a) To produce a result valid below the kinematic thresholds, the integrand is Taylor expanded around the midpoint of the integration region, and integrated over the Feynman parameters. This is all done in FORM [63, 64].

(b) To produce a result valid above thresholds, there is a separate algorithm which determines how to calculate integrals in each kinematic region that is over a threshold. This algorithm is implemented in MathematicA. The subsectors are first mapped onto the complex half plane. The algorithm then determines which configuration to use for each sector, that is, which contour orientation to use for the multiple variable integration and how to partition the subsequent region into smaller pieces. The Taylor expansion and integration are then performed on the new integrands specified by TAYINT. The expansion points are always the midpoints of each interval.

In section 2, each step of the TAYINT algorithm is described in more detail. Section 3 gives an analysis of the virtues of each step of the algorithm, while applications to integrals relevant for phenomenological applications are given in section 4 . Conclusions are drawn in section 5 .

\section{The algorithm}

A generic Feynman loop integral $G$ in an arbitrary number of dimensions $D$ at loop level $L$ with $N$ propagators, wherein the propagators $P_{\tilde{j}}$ with mass $m_{\tilde{j}}$ can be raised to arbitrary powers $\nu_{\tilde{j}}$, has the momentum space representation

$$
\begin{aligned}
G_{\alpha_{1} \ldots \alpha_{R}}^{\mu_{1} \ldots \mu_{R}}(\{p\},\{m\}) & =\left(\prod_{\alpha=1}^{L} \int \mathrm{d}^{D} \kappa_{\alpha}\right) \frac{k_{\alpha_{1}}^{\mu_{1}} \cdots k_{\alpha_{R}}^{\mu_{R}}}{\prod_{\tilde{j}=1}^{N} P_{\tilde{j}}^{\nu_{\tilde{j}}}\left(\{k\},\{p\}, m_{\tilde{j}}^{2}\right)} \\
\mathrm{d}^{D} \kappa_{\alpha} & =\frac{\mu^{4-D}}{i \pi^{\frac{D}{2}}} \mathrm{~d}^{D} k_{\alpha}, \quad P_{\tilde{j}}\left(\{k\},\{p\}, m_{\tilde{j}}^{2}\right)=q_{\tilde{j}}^{2}-m_{\tilde{j}}^{2}+i \delta,
\end{aligned}
$$


where the $q_{j}$ are linear combinations of external momenta $p_{i}$ and loop momenta $k_{\alpha}$. The rank $R$ of the integral is indicated by the number of loop momenta appearing in the numerator and the indices $\alpha_{i}$ denote which of the $L$ loop momenta belongs to which Lorentz index $\mu_{i}$. In what follows, $R=0$ is taken for conciseness, although the TAYINT algorithm is valid for arbitrary rank. The factor of $i \pi^{\frac{D}{2}}$ in $\kappa$ in eq. (2.2) is chosen by convention. The renormalisation scale is denoted by $\mu$, which preserves the dimensionless nature of the coupling constant and is set to unity from here onward. The $+i \delta$ in eq. (2.2) results from the solutions of the field equations in terms of causal Green functions.

For the calculation of unknown integrals, we rewrite every propagator in terms of Feynman parameters $t_{\tilde{j}}$. After integrating the loop momenta, the general form of a scalar Feynman-parameterised multi-loop integral reads

$$
G(\{q\},\{m\})=\frac{(-1)^{N_{\nu}}}{\prod_{\tilde{j}=1}^{N} \Gamma\left(\nu_{\tilde{j}}\right)} \prod_{\tilde{j}=1}^{N} \int_{0}^{\infty} d t_{\tilde{j}} t_{\tilde{j}}^{\nu_{\tilde{j}}-1} \delta\left(1-\sum_{\tilde{l}=1}^{N} t_{\tilde{l}}\right) \frac{\mathcal{U}^{N_{\nu}-(L+1) D / 2}}{\mathcal{F}^{N_{\nu}-L D / 2}(\{q\},\{m\})}
$$

where the functions $\mathcal{U}$ and $\mathcal{F}$ are the first and second Symanzik polynomial, respectively, and are homogeneous in the Feynman parameters.

In order to calculate Feynman parameterised loop integrals as rational functions of the kinematic parameters, the first universal step (U1) in the TAYINT algorithm is to express the given Feynman integral $G$ as a superposition of finite Feynman integrals $G^{\mathrm{F}}$ multiplying factorised poles in $\epsilon$. These finite integrals are either defined in a shifted number of dimensions about $D=4-2 \epsilon$, have propagator powers greater than unity, or both. The combination of these quasi-finite integrals which yields the original integral is found via integration-by-parts $[65,66]$ and Lorentz invariance identities [6] and the Laporta algorithm [67]. In practice, an automated script steers the performance of all necessary steps in the program REDUZE $[59,60]$ towards the generation of the quasi-finite basis. The user must input the integral to be reduced and the integrals that are preferred for the quasifinite basis. In the output, the divergences are restricted to the coefficients of the simpler integrals in the basis, so that the most complicated integral is always finite. Finding an optimal basis partially requires making an educated guess. The guiding principles are to express ultraviolet divergences in terms of vacuum integrals, and to relate subdivergences to sub-graphs of the original integral under consideration.

Once the original Feynman integral has a quasi-finite basis representation, the integrals in this basis are written in terms of their Feynman parametrisation and then decomposed into subsectors which have smoother integrands. These subsector integrals are the building blocks of the rest of the calculation. Their improved smoothness is achieved using sector decomposition [40-43]. Thus, the second universal step (U2) in the TAYINT algorithm is to perform the sector decomposition of the integrals $G^{\mathrm{F}}$ in the quasi-finite basis by passing them to version 3 of the program SECDEC [51]. Therein, the strategy G2, based on refs. [68, 69] and combined with the Cheng-Wu theorem [70, 71] in ref. [51], is used to 
yield sectors of the form

$$
G_{l}^{\mathrm{F}}(\{q\},\{m\})=\prod_{\tilde{j}=2}^{N} \int_{0}^{1} \mathrm{~d} t_{\tilde{j}} t_{\tilde{j}}^{A_{l \tilde{j}}-B_{l \tilde{j}} \epsilon} \frac{\mathcal{U}_{l}^{N_{\nu}-(L+1) D / 2}\left(\vec{t}_{\tilde{j}}\right)}{\mathcal{F}_{l}^{N_{\nu}-L D / 2}\left(\vec{t}_{\tilde{j}},\{q\},\{m\}\right)}, \quad l=1, \ldots, r,
$$

where $r$ is the number of subsector integrals. $A_{l \tilde{j}}$ and $B_{l \tilde{j}}$ are numbers independent of the dimensional regulator $\epsilon$. There are only $N-1$ integrations in total because the first Feynman parameter $t_{1}$ is always integrated out with the $\delta$-distribution. By construction, the deterministic algorithm results in integrands of the type

$$
\begin{aligned}
& \mathcal{U}_{l}=1+u\left(\vec{t}_{\tilde{j}}\right) \\
& \mathcal{F}_{l}=s_{1}+\sum_{\beta} s_{\beta} f_{\beta}\left(\vec{t}_{\tilde{j}}\right),
\end{aligned}
$$

where $u\left(\vec{t}_{\tilde{j}}\right)$ and $f_{\beta}\left(\vec{t}_{\tilde{j}}\right)$ are polynomials in the Feynman parameters $t_{\tilde{j}}$, and $s_{1}, s_{\beta} \in$ $\{\{q\},\{m\}\}$ are kinematic invariants including masses. If the integral were not finite, the singular behaviour would now be contained entirely in the exponents $A_{l \tilde{j}}$ of eq. (2.4). Knowing that the integrals to be computed are finite, a sector decomposition might seem unnecessary. However, it is observed to be vital for an improved convergence of the series expansion.

As the first Feynman parameter has been integrated out, the substitution $t_{\tilde{j}} \rightarrow t_{j}$ is performed to have a sensible hierarchy of parameters, where $j$ runs from 0 to $J-1$. The full integral can then be written in terms of its subsectors

$$
G^{\mathrm{F}}(\{q\},\{m\})=\frac{(-1)^{N_{\nu}}}{\prod_{j=1}^{N} \Gamma\left(\nu_{j}\right)} \Gamma\left(N_{\nu}-L D / 2\right) \sum_{l=1}^{r} G_{l}^{\mathrm{F}}(\{q\},\{m\}) .
$$

For calculations below threshold, there still exist singular behaviours outside of the integration region. Thus the first below-threshold step (BT1) in the TAYINT algorithm is to maximise the distance to the nearest point of non-analyticity and so maximise the accuracy of the expansion. This is acheived by exporting the finite subsector integrands to Mathematica [62] and applying conformal mappings,

$$
t_{j}=\frac{a y_{j}+b}{c y_{j}+d}
$$

In the cases considered so far, for an integrand decomposed into $r$ subsectors and containing $J$ Feynman parameters the optimal mapping has taken the following form,

$$
t_{j}=\left\{\begin{array}{l}
\frac{-y_{j}-1}{y_{j}}, j \in\{0, \ldots J-1\} \text { and } y_{j}, j=J-1 \text { for } l=1 \\
\frac{-y_{j}-1}{y_{j}}, j \in\{0, \ldots J-1\} \text { for } l \in\{2, \ldots, r\} .
\end{array}\right.
$$

For the examples considered, we never mapped the final Feynman parameter in the first sector, as this parameter always appeared in the form $\left(1+t_{J-1}\right)$ in the denominators of the sectors. Thus, it is of no benefit to stretch the surface in that direction. 
The second below-threshold step (BT2) in the TAYINT algorithm is to perform the Taylor expansion of the integrand, the relative simplicity of which is best suited to using FORM [63, 64]. To this end, a FORM procedure was written which Taylor expands functions of the form of the subsector integrands, around any point and to any order.

The third below-threshold step (BT3) is to integrate the $y_{j}$ from $y_{j}(0)$ to $y_{j}(1)$, again in FORM. This yields results for Feynman integrals as rational functions of the kinematics valid everywhere below threshold. The precision is controlled by the order of the expansion.

However, in the kinematic region above the lowest mass threshold of a particular integral, the integrands contain discontinuities on the real axis which prevent a Taylor expansion from converging. Thus, the TAYINT algorithm returns to the result of U2, specifically the multivariate integrands of $G_{l}^{\mathrm{F}}$. These subsector integrands are defined as $\tilde{G}_{l}^{\mathrm{F}}$. The Feynman $+i \delta$ prescription of eq. (2.2) is then implemented in Mathematica. This is done by mapping the multivariate integrands of $G_{l}^{\mathrm{F}}$, onto complex half planes. The TAYINT algorithm then determines the contour configuration which avoids the poles in each kinematic region that is over a threshold. The outline of the over-threshold part of the algorithm, which is implemented in each kinematic region that is above a threshold, is as follows:

1. Implement the Feynman $+\mathrm{i} \delta$ prescription of eq. (2.2) by transforming the sector integrands $\tilde{G}_{l}^{\mathrm{F}}$ as

$$
\tilde{G}_{l}^{\mathrm{F}}\left(t_{j}\right) \rightarrow \tilde{G}_{l}^{\mathrm{F}}\left(t_{j}^{\prime}\right)=\tilde{G}_{l}^{\mathrm{F}}\left(\frac{1}{2}+\frac{1}{2} e^{\mathrm{i} \theta_{j}}\right)
$$

with $j \in\{0, \ldots, J-1\}$. The mapping is chosen such that the real part stays between zero and one and the imaginary part parametrises a contour around a Landau singularity.

2. Find the optimum contour configuration for each $\theta_{j}$ with endpoints 0 and $\pm \pi$, the combination of which is denoted by + or - , respectively. On this contour configuration, find the optimum variable $\theta_{j}^{*}$ to integrate exactly, and hence the optimum post-integration contour configuration, if exact integration is possible.

3. Determine the optimum $n$-fold partitioning $\mathcal{P}_{j}=\left\{(l, h)_{1}, \ldots,(l, h)_{n}\right\}_{j}$ of the integrals in $\theta_{j}$,

$$
\int_{0}^{ \pm \pi} \mathrm{d} \theta_{j}=\sum_{k=1}^{n} \int_{l_{k, j}}^{h_{k, j}} d \theta_{j},
$$

with $h_{n, j}= \pm \pi$ and $l_{1, j}=0$, to use for the Taylor expansion of each sector integrand.

4. Perform the Taylor series expansion in each partition, about the points $e_{j, k}=\frac{1}{2}\left(l_{k, j}+h_{k, j}\right)$ up to order $p$ in each sector

$$
G_{l}^{\mathrm{F}}(\{q\},\{m\}) \approx T_{l}^{\mathrm{F}}(\{q\},\{m\})=T_{l}^{\mathrm{F}(0)}(\{q\},\{m\})+\cdots+T_{l}^{\mathrm{F}(p)}(\{q\},\{m\}),
$$


and estimate the uncertainty of the full result by comparing the relative size of the contribution of the $p$-th order to the full Taylor series expansion, adding the contribution from each $p$-th order expanded sector in quadrature,

$$
\max \left[G^{\mathrm{F}}(\{q\},\{m\})-T^{\mathrm{F}}(\{q\},\{m\})\right]<\frac{\sum_{l=1}^{r}\left(T_{l}^{F(p)}(\{q\},\{m\})\right)^{2}}{\sum_{l=1}^{r} T_{l}^{F}(\{q\},\{m\})} .
$$

Because of the use of exact one-fold integrations where possible, and because of the partitioning of the surface, the over-threshold algorithm combines algebraic and analytic manipulations, requiring flexibility. Therefore, it is implemented in Mathematica.

To elaborate, the first over-threshold step (OT1) is to transform the Feynman parameters of the $r$ subsectors, according to the rule, $t_{j} \rightarrow \frac{1}{2}+\frac{1}{2} \exp \left(\mathrm{i} \theta_{j}\right)$, and also generate a representative sample of the kinematic region in which the results are to be valid. This is a nested list of values for the $\beta$ scales in the integral at $\gamma$ points in the kinematic region within which we desire results, $\mathcal{K}=\left\{\left\{s_{1}, \ldots, s_{\beta}\right\}_{1}, \ldots,\left\{s_{1}, \ldots, s_{\beta}\right\}_{\gamma}\right\}=\left\{\mathcal{K}_{1}, \ldots, \mathcal{K}_{\gamma}\right\}$. After that, the second over-threshold step (OT2) uses the mean absolute value of the $\theta_{j}$ derivatives (MAD: $\bar{m}_{l}$ ), with the kinematic invariants set to the sample values,

$$
\bar{m}_{l}\left(\Theta_{o(0), \ldots, o(J-1)}^{A}\right)=\left.\frac{1}{A} \sum_{a=1}^{A} \frac{1}{\gamma} \sum_{i=1}^{\gamma} \operatorname{Abs}\left[\frac{1}{J} \sum_{j=0}^{J-1}\left(\frac{\partial}{\partial \theta_{j}} \tilde{G}_{l}^{\mathrm{F}}\left(\theta_{j}, \mathcal{K}_{i}\right)\right)\right]\right|_{\left\{\theta_{j}\right\} \rightarrow \Theta_{o(0), \ldots, o(J-1)}^{a}} .
$$

Note that the mean is also taken over the kinematic sample and the points for the $\theta_{j}$ inserted along the surface, $\Theta_{o(0), \ldots, o(J-1)}^{A} \subset \Theta_{o(0), \ldots, o(J-1)}$. The MAD is calculated for all possible $J$-variable complex surfaces in the $\theta_{j}$. These surfaces are the $\Theta_{o(0), \ldots, o(J-1)}$, where $o(j)= \pm$ is the orientation of the $j$ th contour. Each surface is classified by replacing the $\theta_{j}$ variables by $A$ points along it, $\Theta_{o(0), \ldots, o(J-1)}^{A}$, in the mean absolute derivative with respect to the $\theta_{j}$. Scanning the surfaces $\Theta_{o(0), \ldots, o(J-1)}$ using the MAD is done to determine which contour orientation, for example $\Theta_{+-+}$for $J=3$ yields the $\Theta_{o(0), \ldots, o(J-1)}$ best suited for an expansion. The plus and minus signs indicate the direction of motion around the contour. The separation of the points in the $\Theta_{o(0), \ldots, o(J-1)}^{A}$ in the MAD is set to a default value of 0.1 which is sufficient to determine the optimum $\Theta_{o(0), \ldots, o(J-1)}$ surface. However this value can be varied by the user.

The scanning is done in two stages. Firstly, the MAD in the corners of the $\Theta_{o(0), \ldots, o(J-1)}$ surfaces is calculated, as it is here that the change is always the most substantial. Any contour configurations which yield extreme changes at the corners are discarded. Then, the MAD for the remaining $\Theta_{o(0), \ldots, o(J-1)}$ surfaces is calculated, with the corners excluded. This is because the larger changes in the corners mask the changes in the bulk of the $\Theta_{o(0), \ldots, o(J-1)}$ surfaces. The $\Theta_{o(0), \ldots, o(J-1)}$ which minimises the MAD in the second stage is then selected.

As this is a $J$-fold surface, the third over-threshold step (OT3) is to perform all possible one-fold integrations in the $\theta_{j}$ exactly, without using an integrand expansion. A time limit is imposed on this operation. If the limit is exceeded, TAYINT automatically reverts to the $\Theta_{o(0), \ldots, o(J-1)}$ from OT2. 
Next, the fourth over-threshold step (OT4) is to determine the optimum postintegration surface, $\Theta_{o(0), \ldots, o(J-2)}$. To achieve this, all the one-fold exact integrations are performed and all resultant $J-1$ variable integrands examined, using the two-step surface scanning process with the MAD. The $\Theta_{o(0), \ldots, o(J-2)}$ which minimises the MAD is selected. If the mean absolute derivatives of each of the possible $J$ or $J-1$ surfaces are, within a relative difference of 0.01 , equally smooth, then we stop the process. This is because all the possible surfaces are then producing predictions of similar quality and the potential for further optimisation is negligible. Provided there is another $J$ variable contour which has a MAD within a relative difference of 0.01 , the second-best surface is taken and the procedure starts again. The MADs of each surface, with and without exact integration, are finally compared, and the surface with the overall minimum MAD is selected. In the vast majority of cases the post-integration surfaces are chosen, if an exact integration is possible.

The fifth over-threshold step (OT5) determines the optimal way to partition the surface into sections $\mathcal{P}_{j}$ within which the expansions are carried out. The algorithm determines the number of partitions to use and the size of each partition. This is done by using the MAD to calculate the relative size of the fluctuations in each section of the surface. A suitable partitioning is then chosen, i.e., the more fluctuations, the greater the number of partitions, and the denser the fluctuations, the smaller the section enclosing that region of the surface. If no exact integration can be performed, TAYINT can use two more orders or twice as many partitions to maintain the same degree of accuracy.

In summary, the over-threshold part of the algorithm makes a complex mapping in several variables and determines the optimum pre- and post-exact integration contour configuration and the optimum partitions for the integrand expansion. This is done for each kinematic region that is over a thereshold. The resulting integrands of each sector, $\tilde{G}_{l}^{F}\left(\theta_{0}, \ldots, \theta_{J-1}\right)$ are then expanded and integrated in the sixth above-threshold step (OT6),

$$
\begin{aligned}
& T_{l}^{F}(\{q\},\{m\})= \\
& \prod_{j=0}^{J-1}\left(\sum_{k_{j}=1}^{n_{j}} \int_{l_{k_{j}}}^{h_{k_{j}}} \mathrm{~d} \theta_{j} \sum_{s_{j}=0}^{m_{j}} \frac{\left(\theta_{j}-e_{j, k}\right)^{s_{j}}}{s_{j} !} \frac{\partial^{s_{1}+\ldots+s_{J}}}{\partial \theta_{1}^{s_{1}} \ldots \partial \theta_{J-1}^{s_{J}-1}} \tilde{G}_{l}^{F}\left(\{q\},\{m\}, e_{1}, \ldots, e_{J-1}\right)\right),
\end{aligned}
$$

where $e_{j, k}$ are the expansion points, the midpoints of each partition, $m_{j}$ the order of the expansion and $n_{j}$ the number of partitions, in each parameter $\theta_{j}$. The algorithm calculates the Taylor expansion of the integrand and then integrates this expansion to produce an approximation of the integral as a function of the expansion points and integration boundaries. Then all the relevant expansion points and boundaries are inserted to generate the result in each partition. These results are then summed to give the result for each sector. Adding up the results from each sector in each kinematic region generates an expression for the full finite Feynman integral in terms of rational functions of the kinematic scales that is valid everywhere in that region. Thus, systematic approximations are obtained for Feynman integrals with full kinematic dependence, valid in all kinematic regions, above and below mass thresholds. The precision of the approximation is controlled by the order of the expansion and the resolution of the partitioning. 


\begin{tabular}{|l|ll|}
\hline \multicolumn{2}{|l|}{ U1: reduce the Feynman Integral to a quasi finite basis } \\
\hline U2: perform a sector decomposition on the finite integrals in the basis \\
\hline \multicolumn{1}{|c|}{ below-threshold } & \multicolumn{1}{c|}{ over-threshold } \\
\hline BT1: $t_{j} \rightarrow y_{j}$ & OT1: $t_{j} \rightarrow \theta_{j}$, generate $\mathcal{K}$ \\
\hline BT2: $\begin{array}{l}\text { Taylor expand the integrand } \\
\text { and integrate }\end{array}$ & $\begin{array}{lll}\text { OT2: } & \text { find optimum } \\
\Theta_{o(0), \ldots, o(J-1)}\end{array}$ \\
\hline & OT3: & perform one-fold integrations \\
\hline & OT4: $\begin{array}{l}\text { post-integration, find optimum } \\
\Theta_{o(0), \ldots, o(J-2)}\end{array}$ \\
\hline & OT5: $\quad$ determine partition $\mathcal{P}_{j}$ \\
\hline & OT6: & Taylor expand and integrate \\
\hline
\end{tabular}

Table 1. Summary of the individual steps of TAYINT.

Finally, to estimate the uncertainty in the TAYINT calculation, the truncation error in the Taylor expansion is calculated. To do this, the highest order contribution of all $T_{l}^{F}$, where $s_{j}=m_{j}$, are considered, summed in quadrature and divided by the full result, $T^{F}$, where $s_{j}$ runs from 0 to $m_{j}$. Due to the fact that an integration over all $\theta_{j}$ parameters is performed, including the parameter which gives the largest contribution to the uncertainty, all possible sources of uncertainty are taken into account. The resulting uncertainties for each sector are then added in quadrature to produce the final uncertainty estimate in the result. Note that the uncertainty will be overestimated in the vicinity of any kinematical point at which one of the sectors evaluates to a numerical zero by TAYINT, meaning that the $\tilde{G}_{l}^{F}\left(\theta_{0}, \ldots, \theta_{J-1}\right)$ is oscillatory. Nevertheless it always constitutes an overestimation of the uncertainty when less reliable. Moreover, the uncertainty estimate is always highly conservative as the $p$-th order of the Taylor expansion is used to estimate the truncation errors in the results calculated using an expansion up to $p$-th order, rather than the order $p+1$.

A summary of all steps of the TAYINT algorithm is given in table 1.

\section{Discourse on the method}

To facilitate a deeper understanding of the method, the different steps are illustrated for the integrals in figure 1, their characteristics being listed in table 2. The finite sunrise S14 ${ }^{01220}$ [72] and triangle graph T41 [72] serve as examples to illustrate the TAYINT algorithm. The integrals I10 [73], I21 [73], I246 [74] and I39 appear in the two-loop amplitudes $[31,56,75]$ for Higgs-plus-jet production in gluon fusion, mediated through a massive top quark loop. They demonstrate the applicability of TAYINT to complicated multi-loop multi-scale integrals. The number of Feynman parameters quoted in table 2 is counted after performing the integration of the $\delta$-distribution of eq. (2.3). In what follows and where given, the powers of each propagator are denoted by superscripts, i.e. $X^{i j k}$. The kinematic invariants $s$, and $u$ are defined as $s=\left(p_{1}+p_{2}\right)^{2}$ and $u=\left(p_{2}+p_{3}\right)^{2}$, respectively. All momenta are considered incoming. 


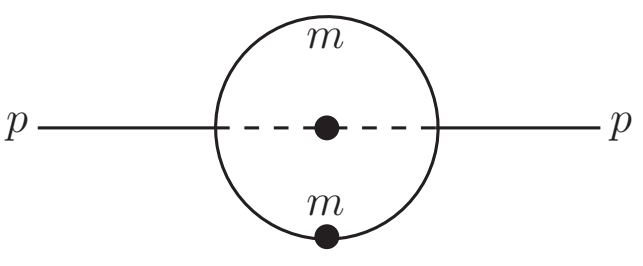

(a) $\mathrm{S} 14^{01220}$

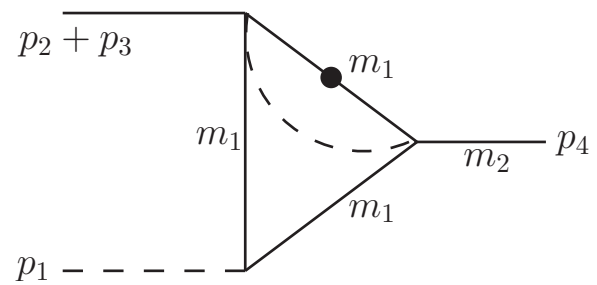

(c) $\mathrm{I} 10$

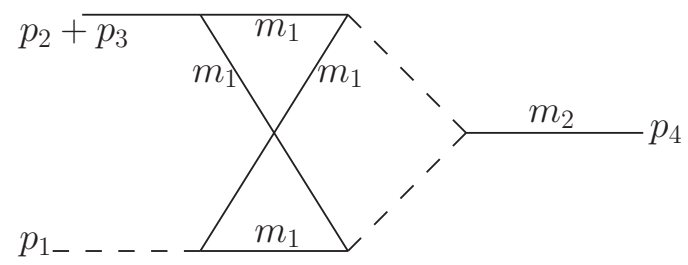

(e) I246

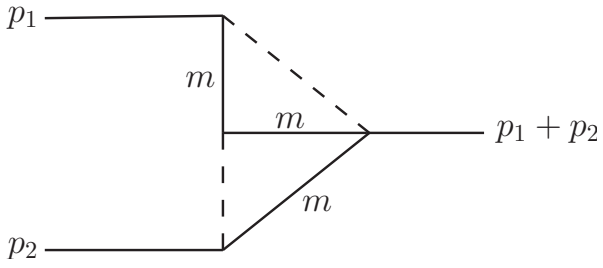

(b) T41

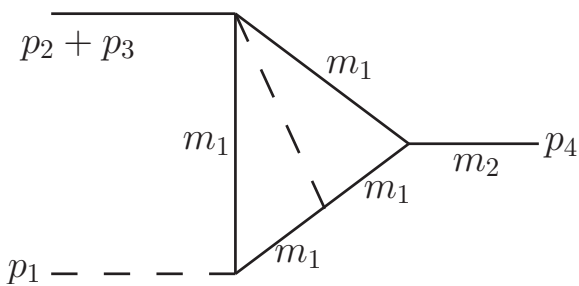

(d) I21

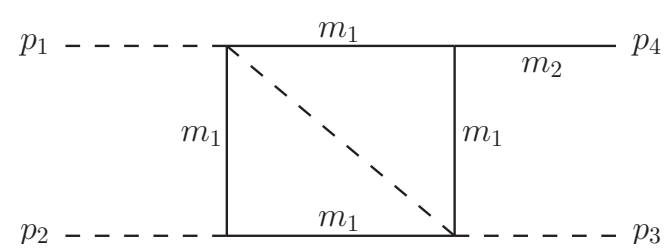

(f) $\mathrm{I} 39$

Figure 1. The two-loop finite sunrise S14 $4^{01220}$ and triangle T41, I10, I21 graphs for which analytical results are available [72, 73]. No fully analytical expressions exist for the non-planar I246, (e), and the box-type integral I39, (f). Dashed lines indicate massless, solid internal lines massive, and dots squared propagators. Solid external lines denote, where indicated, massive and else off-shell particles.

\begin{tabular}{|l|l|l|l|}
\hline Graph & Scales & Feynman parameters & Subsectors \\
\hline S14 $^{01220}$ & 2 & 2 & 3 \\
\hline T14 & 2 & 4 & 28 \\
\hline I10 & 3 & 3 & 8 \\
\hline I21 & 3 & 4 & 16 \\
\hline I39 & 4 & 4 & 16 \\
\hline I246 & 3 & 6 & 36 \\
\hline
\end{tabular}

Table 2. Properties of the integrals corresponding to the diagrams depicted in figure 1. The stated numbers of Feynman parameters correspond to the dimensionality of the expansion and integration steps of TAYINT.

As an example of the step U1 of the TAYINT algorithm, the divergent sunrise integral $\mathrm{S} 14^{01110}[72]$ is written in terms of the finite integrals $\mathrm{S} 14^{01220}$, S14 $4^{01320}$ and the 
tadpole $\mathrm{S} 6^{30300}$,

$$
\begin{aligned}
\mathrm{S} 14^{01110}= & \frac{8 m^{2}\left(p^{2}-4 m^{2}\right)\left(p^{2}+2 m^{2}\right)}{(-3+D)(-8+3 D)(-10+3 D)} \cdot \mathrm{S} 14^{01320} \\
& +\frac{\left((4-D) p^{4}+(-5+D) 8 m^{4}+(18-5 D) 4 p^{2} m^{2}\right)}{(-3+D)(-8+3 D)(-10+3 D)} \cdot \mathrm{S}_{1} 4^{01220} \\
& -\frac{16 m^{4}\left((-4+D) p^{2}+2(-24+7 D) m^{2}\right)}{(-3+D)(-4+D)^{2}(-8+3 D)(-10+3 D)} \cdot \mathrm{S} 6^{30300},
\end{aligned}
$$

where the poles in $\epsilon$ can be seen as the $(-4+D)^{-1}$ terms.

As an example of the TAYINT step U2, the $\mathcal{O}\left(\epsilon^{0}\right)$ coefficient of the first subsector of S14 $4^{01220}$ reads

$$
\mathrm{S} 14_{1}^{01220}=-\prod_{j=0}^{1} \int_{0}^{1} d t_{j} \frac{2}{m^{2}\left(1+t_{0}+t_{0} t_{1}\right)\left(\left(1+t_{1}\right)\left(1+t_{0}+t_{0} t_{1}\right)+t_{0} t_{1} p^{2} / m^{2}\right)},
$$

and has two Feynman parameters, $t_{0}, t_{1}$ and two scales, $p^{2}$ and $m^{2}$. The $\mathcal{O}\left(\epsilon^{0}\right)$ coefficient of the first and second subsector of the I10 [73] integral read

$$
\begin{aligned}
\mathrm{I}_{10}= & \prod_{j=0}^{2} \int_{0}^{1} d t_{j}\left(( 1 + t _ { 0 } + t _ { 1 } + t _ { 0 } t _ { 2 } + t _ { 1 } t _ { 2 } ) \left(-m_{2}^{2} t_{0}-u t_{1}+m_{1}^{2}\left(1+t_{0}^{2}\left(1+t_{2}\right)\right.\right.\right. \\
& \left.\left.\left.+t_{1}^{2}\left(1+t_{2}\right)+t_{1}\left(2+t_{2}\right)+t_{0}\left(2+t_{2}+t_{1}\left(2+2 t_{2}\right)\right)\right)\right)\right)^{-1}, \\
\mathrm{I}_{10} 0_{2}= & \prod_{j=0}^{2} \int_{0}^{1} d t_{j}\left(( 1 + t _ { 0 } + t _ { 1 } + t _ { 2 } + t _ { 1 } t _ { 2 } ) \left(t_{0}\left(-u-m_{2}^{2} t_{1}\right)+m_{1}^{2}\left(1+t_{0}^{2}+t_{2}+t_{1}^{2}\right.\right.\right. \\
& \left.\left.\left(1+t_{2}\right)+t_{1}\left(2+2 t_{2}\right)+t_{0}\left(2+t_{2}+t_{1}\left(2+t_{2}\right)\right)\right)\right)^{-1} .
\end{aligned}
$$

They have three Feynman parameters and three kinematic scales, $m_{1}, m_{2}$ and $u$.

The most precise way of computing the resulting subsector integrals was investigated by comparing the results obtained using various ways of expanding integrands to the literature for the analytically known Feynman integrals. In particular, a comparison of expansions into Taylor series, geometric series, reverse Padé approximations, Chebyshev and Gegenbauer polynomials exposed the Taylor expansion as the most accurate given a variety of test cases. In figure 2, the relative difference between a fifth-order expansion of, and the actual S14 ${ }_{1}^{01220}$ subsector integrand is plotted. While the expansion is rather accurate around the expansion point $\left(t_{1}, t_{2}\right)=\left(\frac{1}{2}, \frac{1}{2}\right)$, the differences between an ordinary Taylor expansion and the actual integrand can become large close to the edges of the integration region. In the case of $\mathrm{S} 14_{1}^{01220}$, these amount to roughly $1 \%$.

The Taylor expansion turns the rational function $R\left(t_{j}\right)$ into a polynomial $P\left(t_{j}\right)$ which can be integrated analytically. Thus, it is necessary to check that accurate results for integrals can still be obtained after expanding the integrand. To this end, the example integral T41, the kinematic dependence of which is parameterised entirely by the dimensionless ratio $x$,

$$
x=\frac{\sqrt{s+4 m^{2}}-\sqrt{s}}{\sqrt{s+4 m^{2}}+\sqrt{s}},
$$




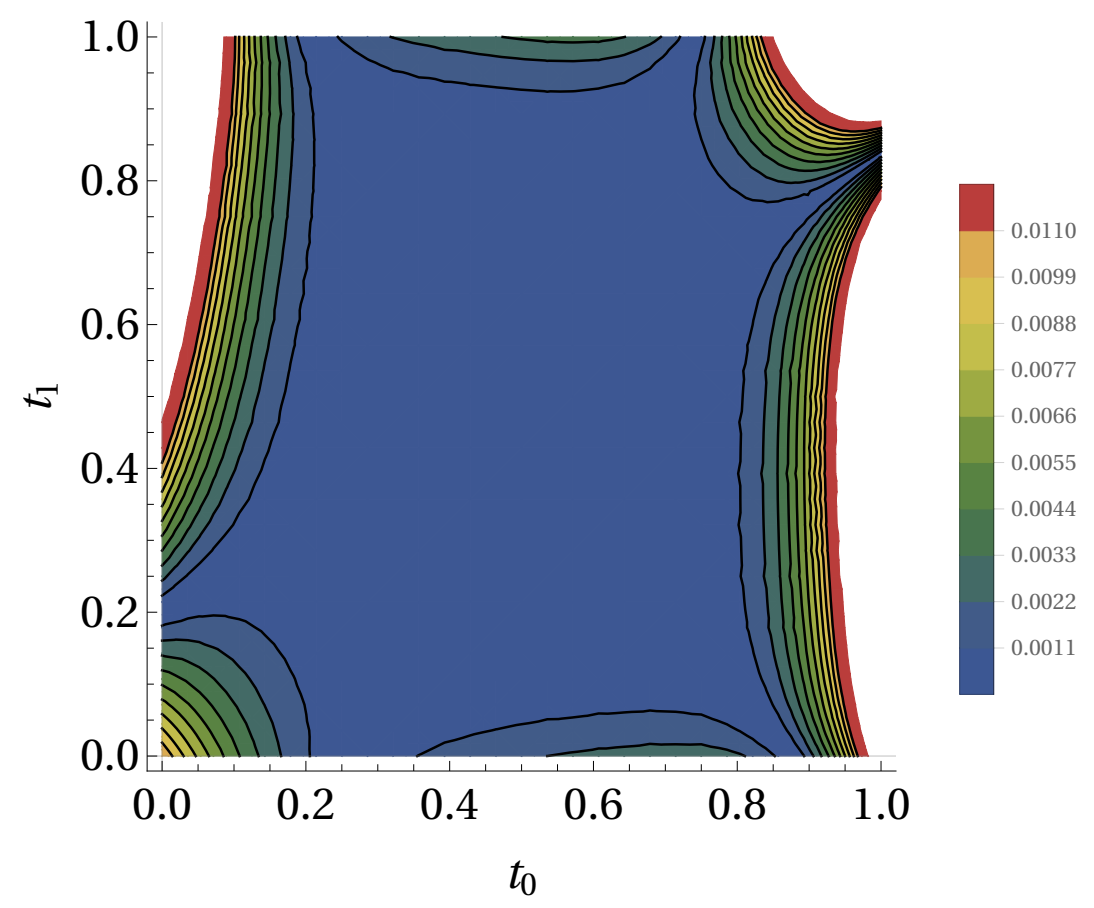

Figure 2. A contour plot of the relative difference between an ordinary sixth-order Taylor expansion and the actual integrand of $\mathrm{S} 14_{1}^{01220}$. The Taylor expansion is around the point $\left(t_{1}, t_{2}\right)=\left(\frac{1}{2}, \frac{1}{2}\right)$ and $p^{2}=-\frac{1}{2} m^{2}, m^{2}=20000 \mathrm{GeV}^{2}$.

was calculated by Taylor expanding the integrand to sixth order. In figure 3(a), the result is compared to the exact result of ref. [72], with the truncation error of the Taylor expansion shown in the lower half of the plot. The Taylor-obtained result is plotted as a solid blue line in contrast to the literature result in a red dot-dash line. The sixth-order truncation error is plotted below using a lilac band. It can be observed that the combination of expansion and integration is effective for calculating Feynman integrals via their subsectors for most values of $x$.

For $x \approx 100$, the discrepancy between approximated and exact result roughly reaches an unacceptable $9 \%$.

To improve on the quality of the approximation, methods to maximise the distance to the nearest point of non-analyticity were investigated. The underlying rationale is that the convergence radius of a Taylor expansion is limited by the distance from the expansion point to the nearest point of non-analyticity. The latter are found in the region $t_{j} \in[0,-\infty]$ for the subsectors. The use of conformal mappings in the $t_{j}$, as detailed in eqs. (2.8) and (2.9), maximises the convergence radius for the examples considered. As an example, the effect of a conformal mapping at the integrand level can be seen in figure 4. In the upper plot, the integrand of integral $\mathrm{I}_{10}$ with two Feynman parameters set to zero,

$$
\mathrm{I}_{10}\left(t_{0}, t_{1}=0, t_{2}=0\right)=\prod_{j=0}^{2} \int_{0}^{1} d t_{j} \frac{1}{\left(1+t_{0}\right)\left(-m_{2}^{2} t_{0}+m_{1}^{2}\left(1+2 t_{0}+t_{0}^{2}\right)\right)},
$$




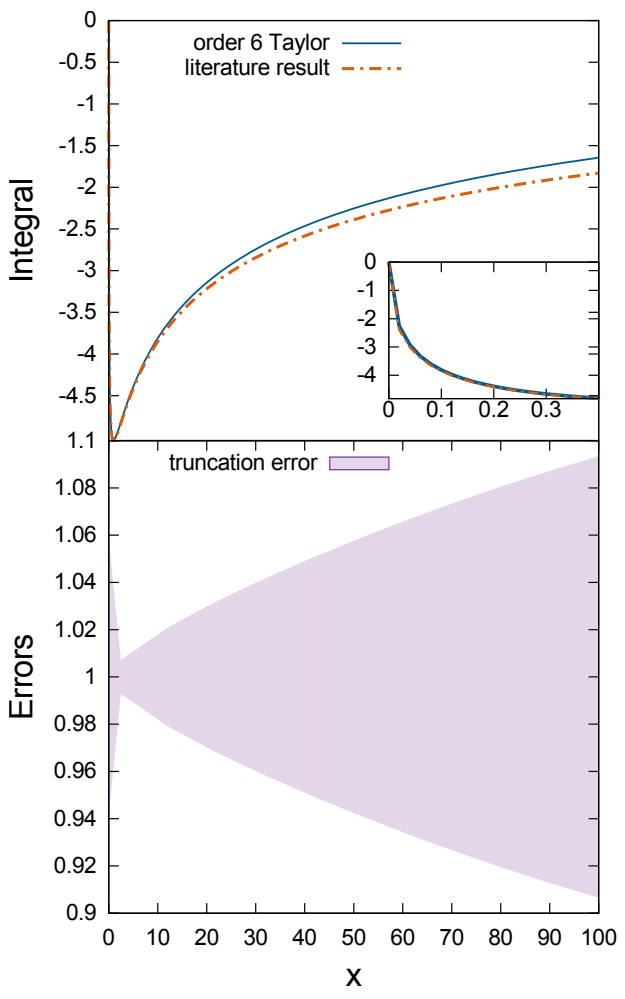

(a)

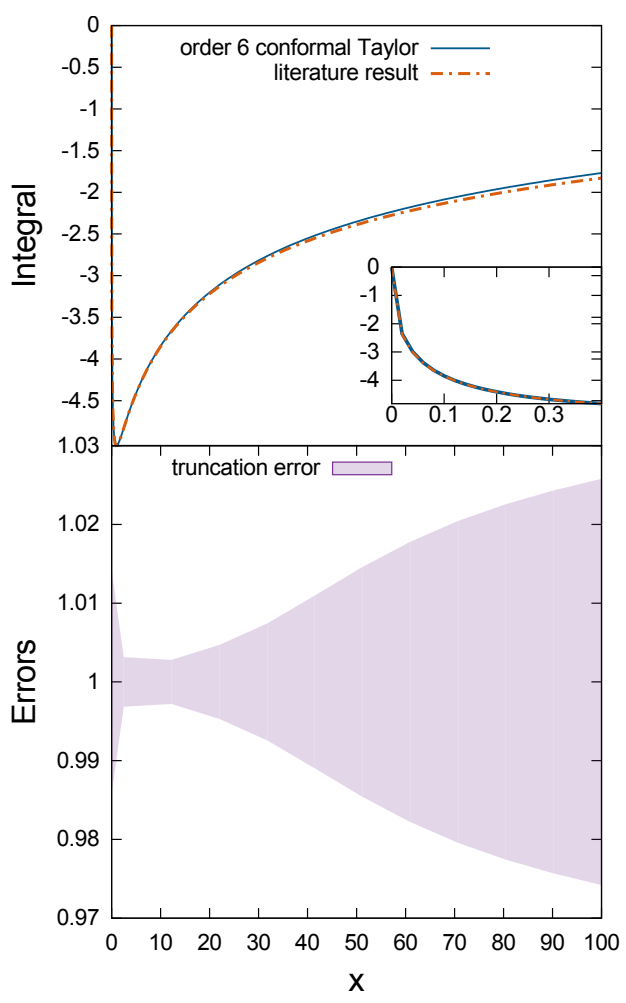

(b)

Figure 3. A comparison between the integrated approximated result for the $\mathcal{O}\left(\epsilon^{0}\right)$ coefficient of T41 and the analytic result, using 3(a) an ordinary Taylor expansion on the integrand, and 3(b) a Taylor expansion enhanced by a conformal mapping. The inlay figures have the same axis labels as the larger plots.

is shown. It has a point of non-analyticity outside the integration region, at $t_{0}=-1$. Applying the conformal mapping,

$$
t_{0}=\frac{-1-y_{0}}{y_{0}}
$$

on the integrand, as detailed in eq. (2.9), the region of integration changes to $y_{0} \in\left[-1,-\frac{1}{2}\right]$ and the non-analytic point is stretched to infinity, as shown in the lower plot of figure 4 . The plot insets zoom into the actual region of integration.

The quantitative improvement can be seen by comparing figures 3(a) and 3(b). In figure 3(a), the sixth-order Taylor expanded result for the $\mathcal{O}\left(\epsilon^{0}\right)$ coefficient of the T41 integral is compared to the exact result known from the literature [32]. In figure 3(b), a conformal mapping is applied to the T41 integrand before performing a Taylor expansion up to sixth order. For $x \approx 100$, the discrepancy between approximated and exact result decreases to less than $3 \%$ when using a conformal mapping. The inset plot shows the behaviour around the threshold in $s$. 

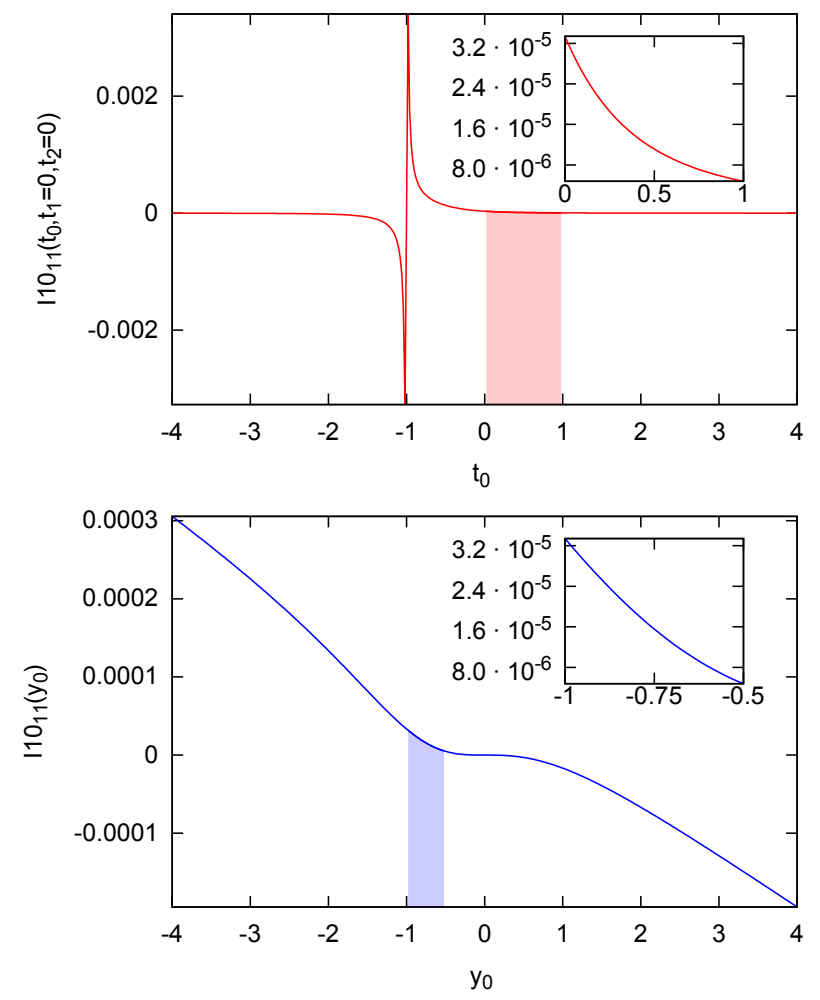

Figure 4. Plot of the one dimensional integrand of eq. (3.6), before and after the conformal mapping. The integration region is shaded and also shown in the inset plots. The masses are set to $m_{1}=173 \mathrm{GeV}$ and $m_{2}=\frac{m_{1}}{\sqrt{2}}$.

To view the effect of applying a conformal mapping to a more complicated example, the ratio of the result computed with an integrand Taylor expansion up to sixth order and the result computed numerically using SECDEC is plotted for the $\mathcal{O}\left(\epsilon^{0}\right)$ coefficient of the full integral I10, see figure 5. Error bars on the numerical results from SECDEC are not plotted due to the high requested numerical accuracy of $10^{-8}$. The ratio is plotted over a kinematic range below threshold, the result with a conformal mapping shown in green and the one without mapping in blue. The mean ratio between SECDEC and the TAYINT result over the plotted range is 1.00043 with the conformal mapping, and 1.00134 without it. Using the conformal mapping therefore increases the precision by more than a factor of three.

No further steps are required to calculate Feynman integrals below threshold. However, above thresholds there are integrable singularities in the subsectors, within the region of integration, which renders all steps beyond the sector decomposition moot. By transforming to the complex plane (OT1), these singularities can be avoided. In figure 6 a slice of $\mathrm{I}_{2}$ is plotted in $t_{0}$ without, and in $\theta_{0}$ with, a complex mapping, respectively. In figure $6(\mathrm{a})$, the integrand without an analytical continuation to the complex plane contains a series of threshold singularities. The ridges are cut for better comparison with figure 6(b). The 


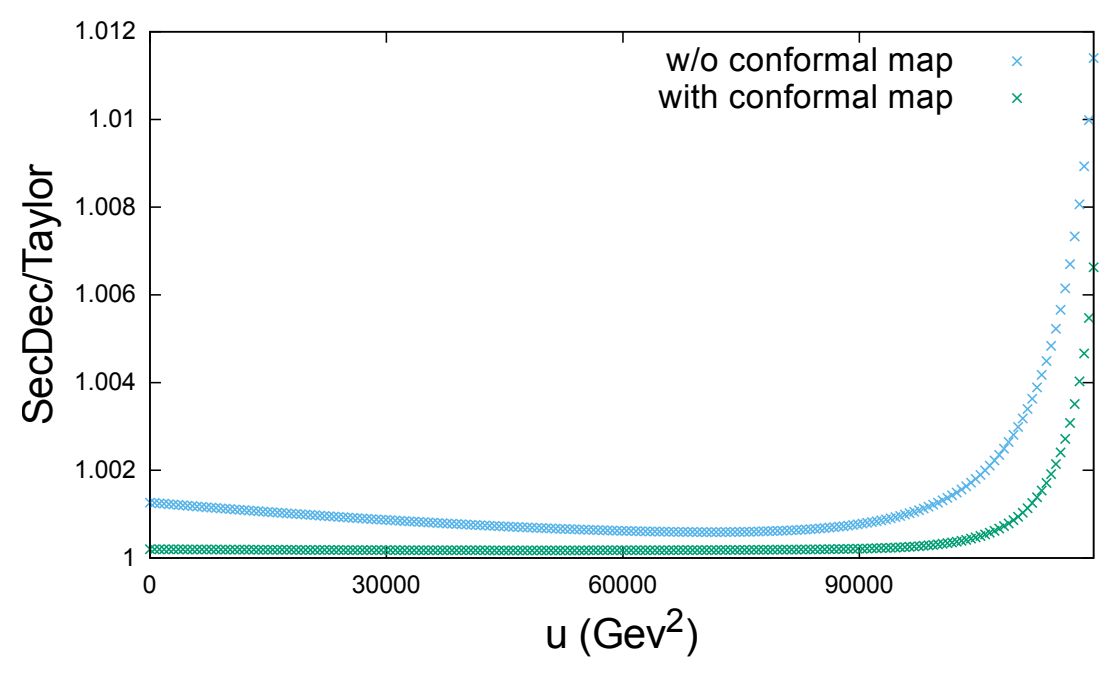

Figure 5. The ratio of the SECDEC result and an ordinary Taylor expansion (sixth-order) are shown with (green) and without (blue) conformal mapping, for the $\mathcal{O}\left(\epsilon^{0}\right)$ coefficent of the integral I10. The scale $u$ is below the $4 m_{1}^{2}$ threshold and $m_{2}=\frac{m_{1}}{\sqrt{2}}, m_{1}=173 \mathrm{GeV}$.

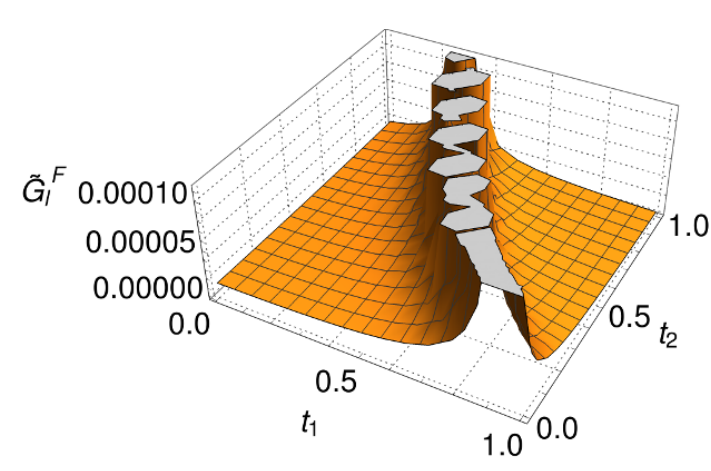

(a)

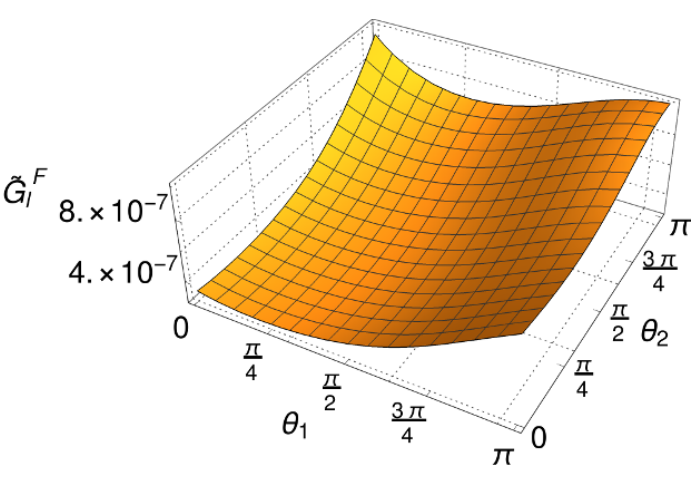

(b)

Figure 6. A slice of the $\mathrm{I}_{1} 0_{2}$ integrand at $\mathcal{O}\left(\epsilon^{0}\right)$ (a) without a complex mapping and (b) with a complex mapping and the contour orientation determined via TAYINT, setting $\theta_{0}=\frac{\pi}{2}, u=5.44 m_{1}^{2}$, $m_{2}=\frac{m_{1}}{\sqrt{2}}$ and $m_{1}=173 \mathrm{GeV}$. In (a) no reorientation of contours is possible as the surface is constrained to the real line.

complex integrand in figure 6(b) shows a smooth behaviour everywhere in the integration region. This demonstrates how integrable poles in the physical region can be avoided and confirms that an ordinary series expansion of the integrand in the Feynman parameters $t_{j}$ is not sufficient to reproduce the actual result.

OT1 yields a version of the subsectors which can take different forms depending on the configuration of the complex contours. Thus, the optimum contour configuration from the possible $\Theta_{o(0), \ldots o(J-1)}$ surfaces must be determined. So must the optimum variable to integrate exactly, $\theta_{j}^{*}$, if exact integration is possible. This yields the optimum postintegration surface, $\Theta_{o(0), \ldots, o(J-2)}$. Finding these optimum configurations is done in steps 


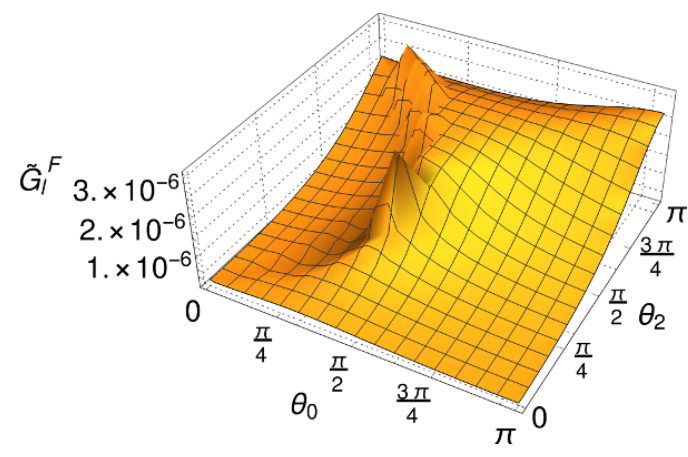

(a)

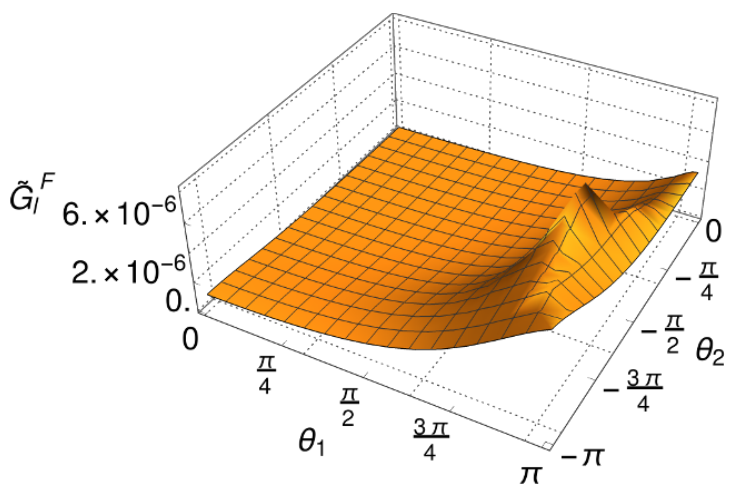

(b)

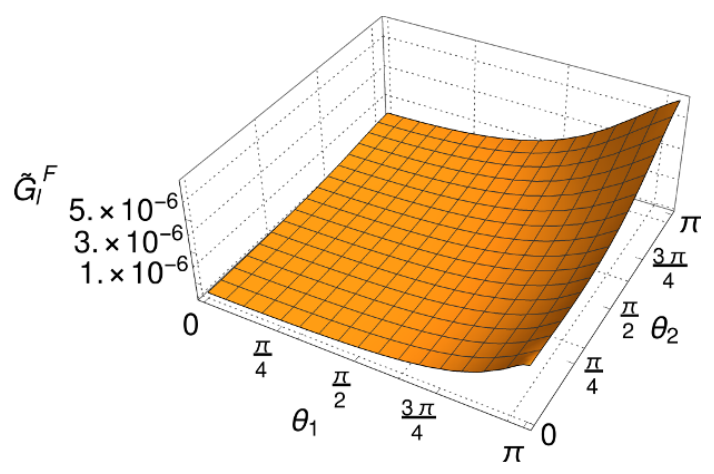

(c)

Figure 7. The $\mathrm{I}_{1} 0_{2}$ integrand at $\mathcal{O}\left(\epsilon^{0}\right)$ is shown after a complex mapping and exact integration of one variable in three different configurations. In (a), the contour orientation is chosen by the algorithm but an arbitrary choice of integration variable is allowed. In (b), the exact integration variable is chosen by the algorithm but an arbitrary choice of contour is allowed. In (c), the contour and exact integration variable are chosen by TAYINT. The kinematic scales are set to $u=5.44 m_{1}^{2}$, $m_{2}=\frac{m_{1}}{\sqrt{2}}$ and $m_{1}=173 \mathrm{GeV}$.

OT2-4. In figure 7, the integrand of $\mathrm{I}_{2}$ is plotted, with one integration performed exactly. There exist many possible pre- and post-integration contours. Not all of these contours are suitable for a Taylor expansion of the integrand. This is because, along the unsuitable contours, the integrand contains non-analytic structures within the region of integration. If such a contour was chosen, the algebraic result for that sector would not always converge at all kinematic points. The TAYINT algorithm avoids these and selects a pre- and postintegration contour configuration which yields a smoothly behaved integrand, which has a well defined Taylor expansion. Figures $7(\mathrm{a})$ and $7(\mathrm{~b})$ illustrate this. The optimal result is achieved when both contours are determined by TAYINT, see figure $7(\mathrm{c})$.

After the analytic continuation of the subsector integrands is optimised, large gradients along the edges of the complex surfaces, see e.g. the integrand surface along $\theta_{0}=\pi$ of figure $7(\mathrm{c})$, are addressed in OT5. To maximise the precision of the result, the surfaces are partitioned and expansions performed around the central value of each partition. The 


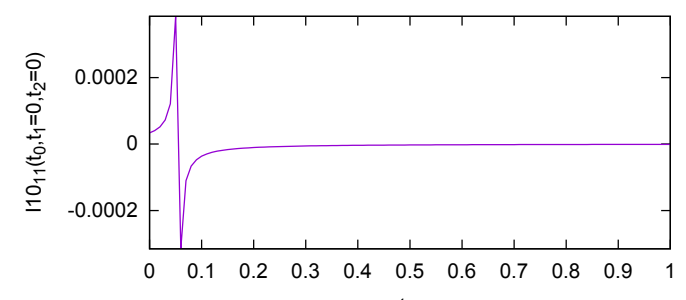

$t_{0}$

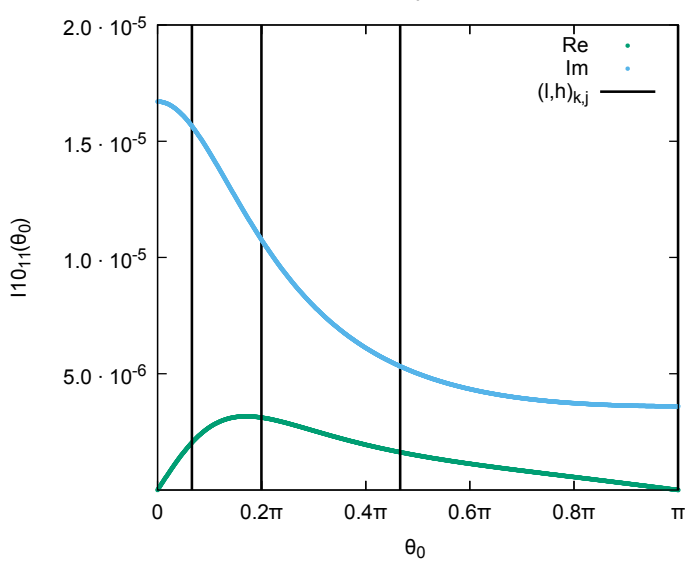

Figure 8. Plot of the one dimensional integrand of eq. (3.6), with chosen values $m_{2}=781.25 \mathrm{GeV}$ and $m_{1}=173 \mathrm{GeV}$. The upper plot shows the integrand without, the lower plot shows the integrand with the implementation of the Feynman $+\mathrm{i} \delta$ prescription. The partitioning of the integral according to eq. (2.11) is illustrated by the black lines.

Taylor expanded sections are then integrated and combined to yield a result for the entire sector. The rationale behind the partitioning is demonstrated in figure 8 , where the one-dimensional integrand of eq. (3.6) is shown for kinematic invariants set to arbitrarily chosen over-threshold values $m_{2}=781.249 \mathrm{GeV}$ and $m_{1}=173 \mathrm{GeV}$. In the upper plot, a discontinuity along the real line arising from the missing implementation of an analytic continuation of the integrand into the complex plane can be observed. To remedy this problem, the integrand is analytically continued into the complex plane, as described in eq. (2.10) and demonstrated by showing the transformed real and imaginary part of the integrand in the lower plot of figure 8, in green and blue, respectively. Even though the integrand is now suited to a Taylor expansion, the gradient of the integrand is large for $\theta_{0} \rightarrow 0$. Thus, the integration region is split according to eq. (2.11), with the new integration boundaries $(l, h)_{k, j}$ marked by black lines in the bottom plot of figure 8 . The expansion and integration is performed in each partition individually. The algorithm splits the integral such that within each partition the gradient is small. Hence, the convergence of the Taylor expansion within each integral piece is faster than that of an expansion of the whole integrand.

For generating results valid above thresholds, one might ask why an ordinary Taylor expansion cannot be used after steps OT1-4. The importance of a partitioning is illustrated for the I10 diagram in figure 9. The result generated with a Taylor expansion without 

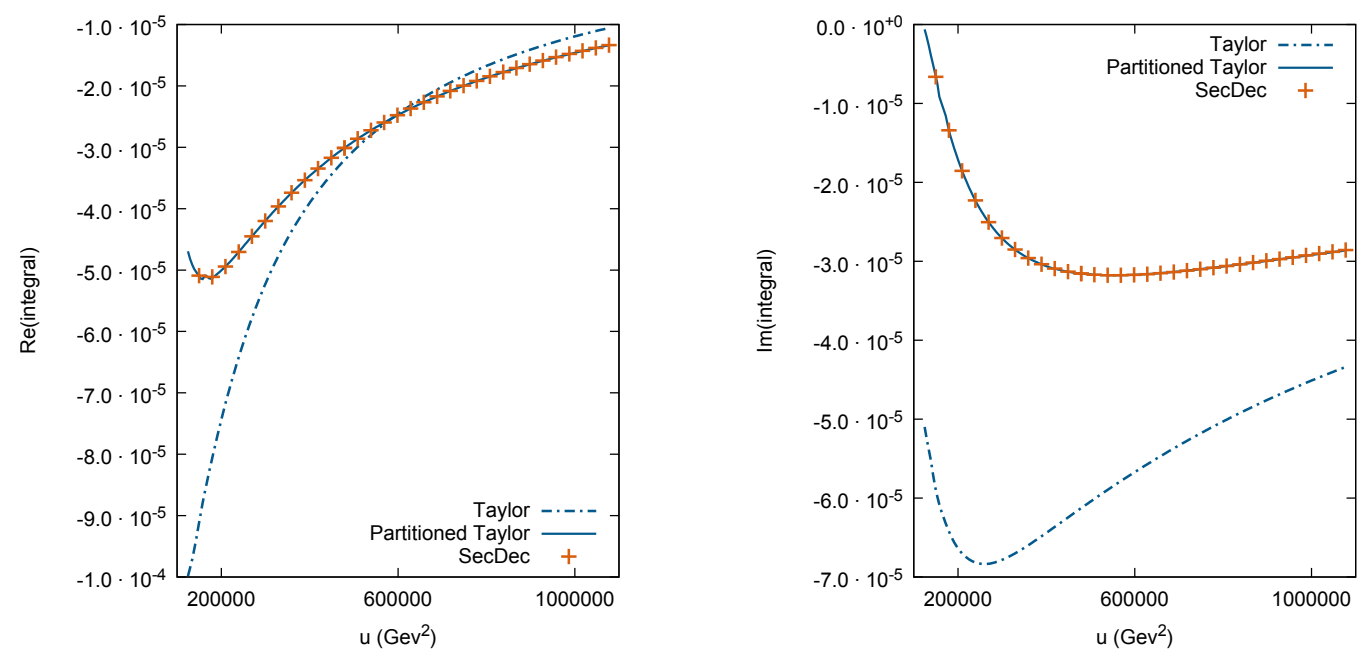

Figure 9. I10 integral at $\mathcal{O}\left(\epsilon^{0}\right)$ over the $4 m_{1}^{2}$ threshold with and without partitions. The kinematic scales are $m_{2}=\frac{m_{1}}{\sqrt{2}}$ and $m_{1}=173 \mathrm{GeV}$.

partitions is plotted as a dot-dashed blue line, the result obtained with partitions is shown as a solid blue line and the SECDEC points are orange crosses. Without using partitions, the Taylor result converges slowly and a huge number of orders in the expansion would be required. But if a particular integrand is extremely complicated then there will be a limit on the order to which the Taylor expansion can be computed before intermediate expressions in FORM or MAThematica become too large for the expansion to be completed. For the subsectors of I10, with each increase in the order of the Taylor expansion the intermediate expressions in TAYINT increase in size by a factor of three. To put this into context, it is instructive to take a closer look at the first subsector of the integral I39,

$$
\begin{aligned}
\mathrm{I} 39_{1}= & \prod_{j=0}^{3} \int_{0}^{1} d t_{j}\left(\left(1+t_{0}+t_{1}+t_{2}\right)\left(1+t_{0}+t_{1}+t_{2}+\left(1+t_{0}\right)\left(t_{1}+t_{2}\right) t_{3}\right)\right. \\
& \left.\left(1+t_{0}+t_{1}+t_{2}+\left(1+t_{0}\right)\left(t_{1}+t_{2}\right) t_{3}\right) m_{1}^{2}-t_{0} t_{2} u-t_{1}\left(s+t_{0} m_{2}^{2}\right)\right)^{-1} .
\end{aligned}
$$

All subsectors of I39 have four Feynman parameters and four scales, $s, u, m_{1}$ and $m_{2}$. Because of the complexity of these sectors after performing the first integration exactly, an expansion beyond (typically) tenth-order in the Taylor series is not possible beyond $\mathcal{O}\left(\epsilon^{1}\right)$ over threshold, due to the size of the algebraic expressions that are generated at intermediate stages. However, the result can still be made as precise as required by using more partitions. It is important to notice that the increase in the number of partitions allows the circumvention of memory bottlenecks. The expressions for each partition can be truncated at a lower order in the Taylor series than the full expression, owing to the smaller distance from the expansion point. Increasing the number of partitions does increase the algebraic computation time required to obtain the series expansion, which can however be parallelised trivially. Once the result is computed, an instant evaluation at arbitrary phase 


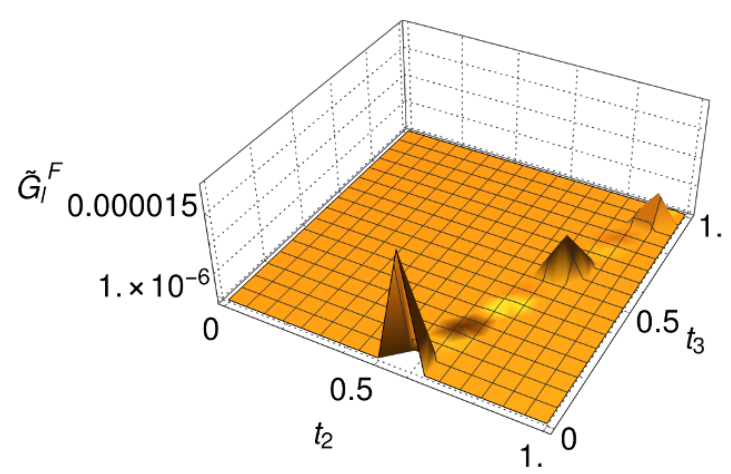

(a)

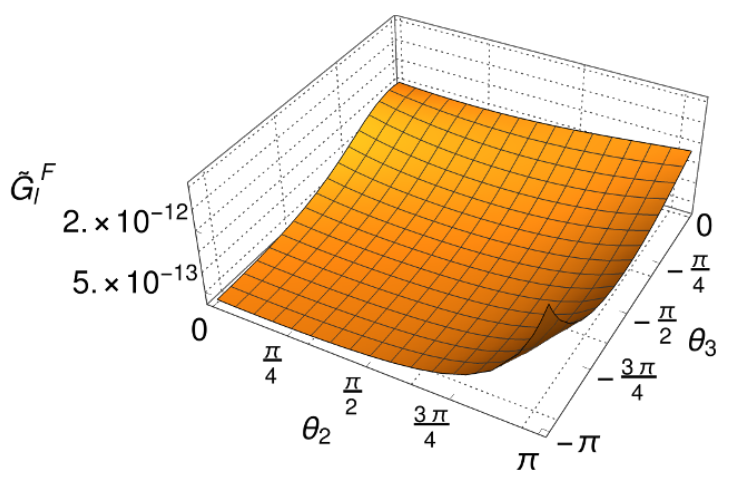

(b)

Figure 10. A slice of the absolute value of the $\mathrm{I} 246_{1}$ integrand at $\mathcal{O}\left(\epsilon^{0}\right)$ in the first over-threshold region (a) without a complex mapping, $t_{0}=1, t_{1}=\frac{1}{10}, t_{4}=\frac{1}{10}, t_{5}=0$ and (b) with a complex mapping, $\theta_{0}=-\pi, \theta_{1}=-\frac{\pi}{10}, \theta_{4}=-\frac{\pi}{10}, \theta_{5}=0$, and the contour orientation determined via TAYINT, setting $u=3.2 m_{1}^{2}, m_{2}=\frac{m_{1}}{\sqrt{2}}$ and $m_{1}=173 \mathrm{GeV}$.

space points is possible. Thus, an increased partitioning enables the result to meet a target precision. For example, the I10 sectors have three Feynman parameters and three scales, and the Taylor series can be computed to beyond tenth order. Doubling the number of partitions at sixth order reduces the error in the real part by $91 \%$ and that of the imaginary part by $86 \%$.

The over-threshold part of TAYINT is implemented in each kinematic region that is over a mass threshold. To illustrate this in the case of multiple thresholds, we consider the integrand of one specific sector of the integral I246, figure 1(e), which we denote as I246 ${ }_{1}$. This integrand is plotted prior to running the TAYINT algorithm, and using the TAYINT algorithm to determine the contour configuration, over the first threshold in figure 10, and over the second threshold in figure 11. In figure 10(a), the integrand is plotted in terms of the (undeformed) Feynman parameters and contains threshold singularities. The complex integrand shown in terms of the deformed variables in figure 10(b) manifests smooth behaviour throughout the integration region. Likewise, in figure 11(a), the integrand in terms of the undeformed Feynman parameters contains more threshold singularities, but the complex integrand in figure 11(b) still manifests smooth behaviour throughout the integration region. Thus in both regions over thresholds TAYINT can take integrands with threshold singularities and convert them into smooth integrands which can be calculated algebraically by means of a Taylor expansion, to produce results valid everywhere in each threshold region. Moreover, the TAYINT results for individual sectors of I246 have been checked against the corresponding SECDEC results at a kinematic point above the first threshold in $u$, on the transitional point between the first and the second threshold, and above the second threshold, explicitly $u=47886.4 \mathrm{GeV}^{2}, 119716 \mathrm{GeV}^{2}, 146053.52 \mathrm{GeV}^{2}$. The TAYInT and SECDEC results are in agreement. 


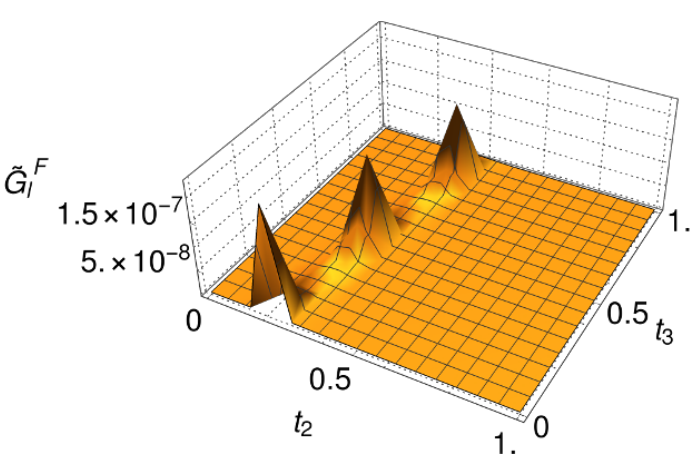

(a)

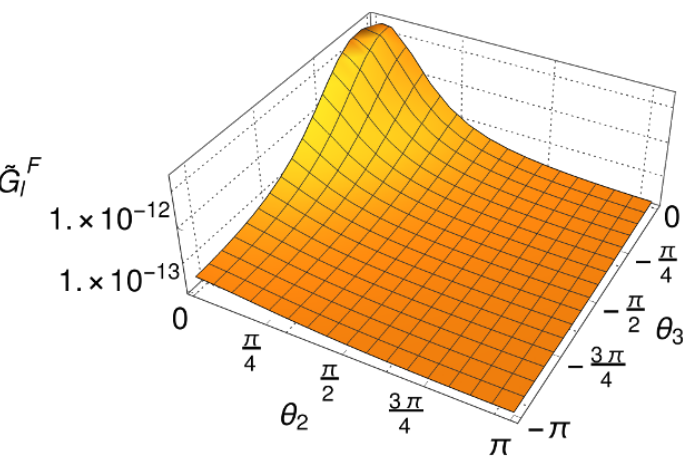

(b)

Figure 11. A slice of the absolute value of the I246 ${ }_{1}$ integrand at $\mathcal{O}\left(\epsilon^{0}\right)$ in the second over-threshold region (a) without a complex mapping, $t_{0}=1, t_{1}=\frac{1}{10}, t_{4}=\frac{1}{10}, t_{5}=0$ and (b) with a complex mapping, $\theta_{0}=-\pi, \theta_{1}=-\frac{\pi}{10}, \theta_{4}=-\frac{\pi}{10}, \theta_{5}=0$, and the contour orientation determined via TAYINT, setting $u=7.2 m_{1}^{2}, m_{2}=\frac{m_{1}}{\sqrt{2}}$ and $m_{1}=173 \mathrm{GeV}$.

\section{Application to three-scale two-loop four-point integrals}

To illustrate the power of TAYINT, explicit results for the integrals I10 and I39 are presented below and above threshold, and for different orders in $\epsilon$, respectively.

In figure 12, results for the finite I10 integral below threshold and up to $\mathcal{O}\left(\epsilon^{2}\right)$ are shown. In the upper half of the plots, the approximated TAYINT result is shown as a blue solid line, overlaid with results generated with the program SECDEC, depicted as orange crosses. The only, though hardly noticeable, deviation can be seen directly on and around the threshold, at $u=4 m_{1}^{2}$, where the difference of the TAYINT with respect to the exact result reaches at most $0.7 \%$. In the lower half of the plots, the uncertainty band of TAYINT is shown in lilac and can be compared to the uncertainties coming from SECDEC shown in green. The SECDEC results were computed using default numerical integration parameters and the integrator VEGAS [76], asking for a relative accuracy of $10^{-3}$. The relative accuracy is adapted to the accuracy of the TAYINT results. The same colour coding is used for all subsequent plots of results.

There is no appreciable precision loss as the order in $\epsilon$ increases. For I10 the mean $\frac{\text { SECDEC }}{\text { TAYINT }}$ ratios are 1.0006, 1.00039, 1.00021 at $\epsilon^{0}, \epsilon^{1}$ and $\epsilon^{2}$, respectively.

In figure 13, results for the finite I39 integral up to $\mathcal{O}\left(\epsilon^{2}\right)$ are plotted in $s$ below threshold, asking for a relative SECDEC accuracy of $10^{-3}$. Again, the maximal deviation between the TAYINT and SECDEC results can be found on the threshold of $s=4 \mathrm{~m}_{1}^{2}$ at $\mathcal{O}\left(\epsilon^{0}\right)$, where the difference reaches $0.1 \%$, rounded up. There is no appreciable precision loss as the order in $\epsilon$ increases. In fact, quite the contrary, the mean $\frac{\text { SECDEC }}{\text { TAYINT }}$ ratios are $1.0003,1.00017,1.00009$ at $\epsilon^{0}, \epsilon^{1}, \epsilon^{2}$ respectively.

In figure 14 the TAYINT result for I10 at $\mathcal{O}\left(\epsilon^{0}\right)$, obtained with a sixth-order, fourfold partitioned, Taylor expansion, is plotted and compared to results from the program SecDec. The SEcDec results were computed using default numerical integration parameters and the integrator VEGAS, asking for a relative accuracy of $10^{-4}$. The plot shows 


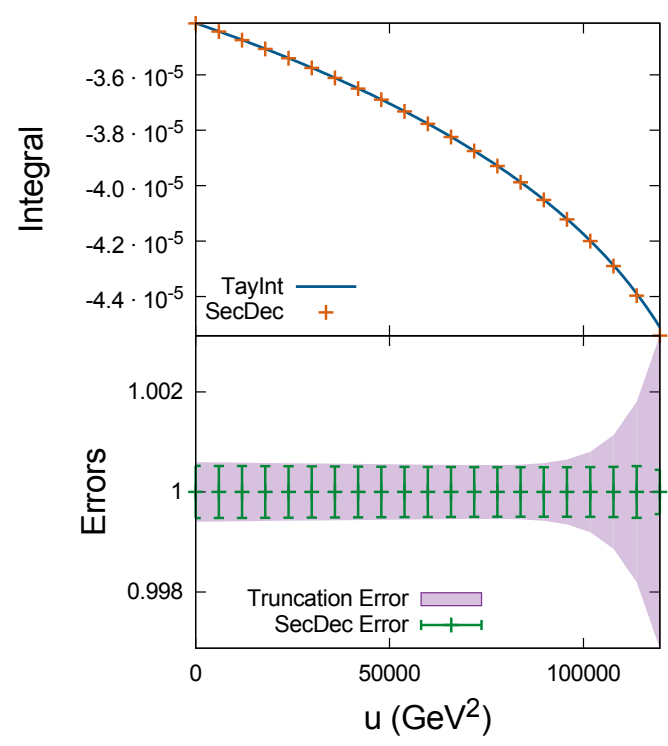

(a)

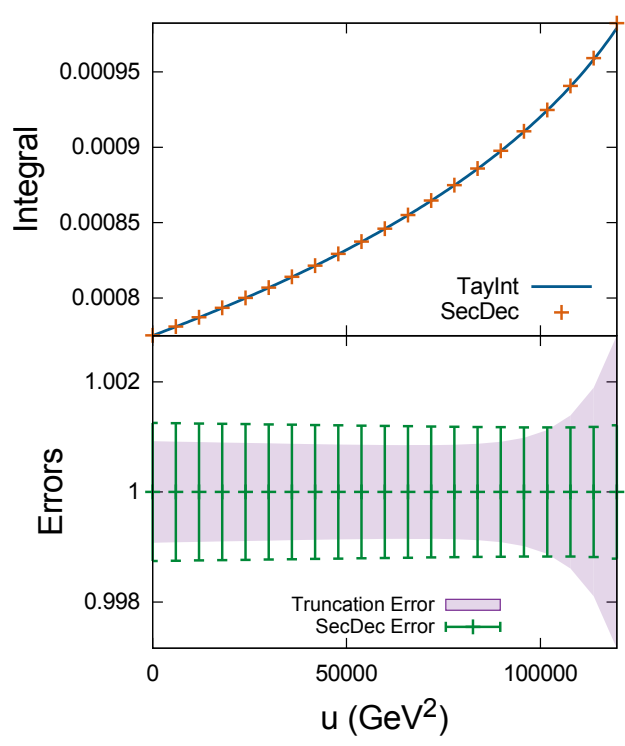

(b)

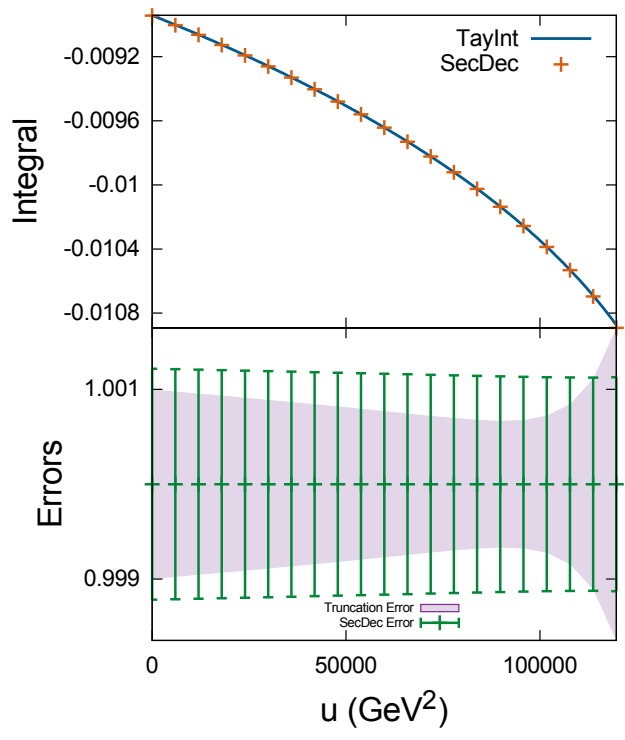

(c)

Figure 12. I10 below threshold, calculated with an eighth-order series expansion at; (a) $\mathcal{O}\left(\epsilon^{0}\right)$, (b) $\mathcal{O}\left(\epsilon^{1}\right)$, (c) $\mathcal{O}\left(\epsilon^{2}\right)$ with $m_{2}=\frac{1}{\sqrt{2}} m_{1}$ and $m_{1}=173 \mathrm{GeV}$. The lower plots show the relative uncertainties of the TAYINT and numerical SECDEC results, respectively.

the dependence on the scale $u$ in the threshold region around $u=4 m_{1}^{2} \sim 120000 \mathrm{GeV}^{2}$ and above the threshold. By referring to figure 12(a), a smooth transition from the belowthreshold expansion to the over-threshold expansion can be observed. The size of the error directly on threshold is rooted in the fact that it displays a Landau singularity, a physical 


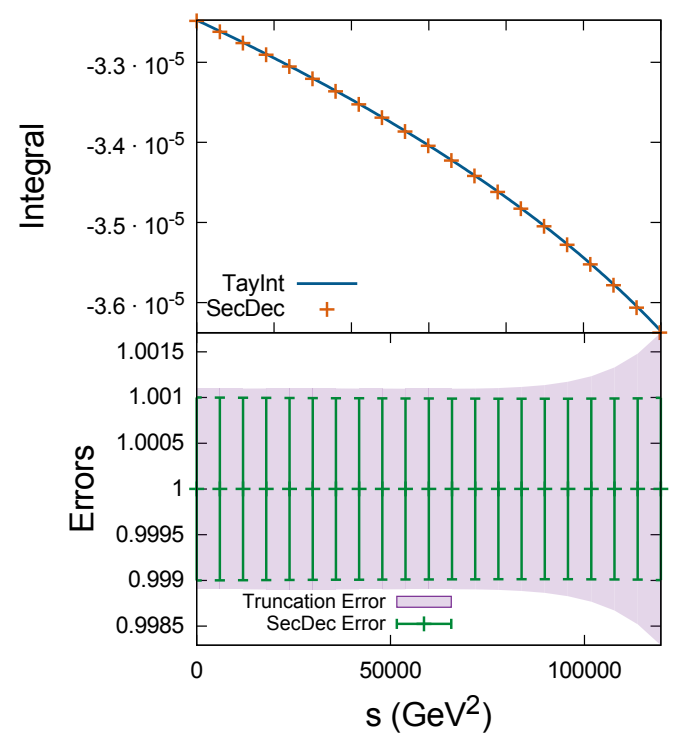

(a)

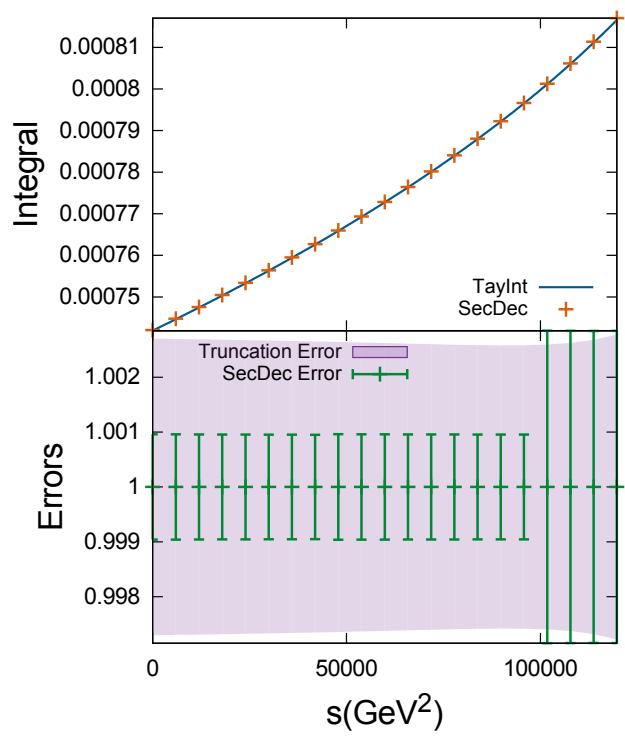

(b)

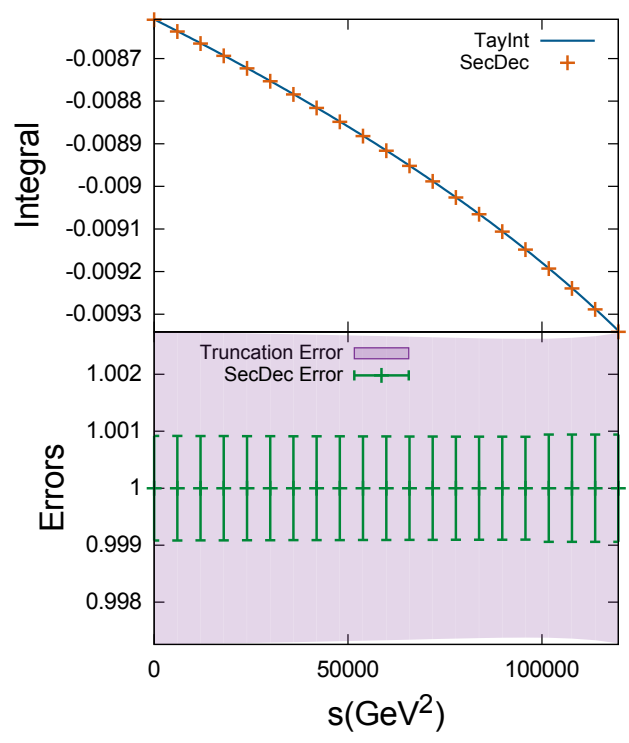

(c)

Figure 13. I39 below threshold, calculated with a sixth-order series expansion at; (a) $\mathcal{O}\left(\epsilon^{0}\right)$, (b) $\mathcal{O}\left(\epsilon^{1}\right),\left(\right.$ c) $\mathcal{O}\left(\epsilon^{2}\right)$ with $u=-59858 \mathrm{GeV}^{2}, m_{2}=\frac{1}{\sqrt{2}} m_{1}$ and $m_{1}=173 \mathrm{GeV}$. The lower plots show the relative uncertainties of the TAYINT and numerical SECDEC results, respectively.

discontinuity, where the function is no longer holomorphic and the Taylor series has zero radius of convergence. Hence a Taylor series expansion has to break down by construction. Nonetheless, even on the threshold, the relative accuracy of the TAYINT result only drops to $10^{-2}$. To generate the SECDEC results for figures 14 and 15 a relative accuracy of $10^{-4}$, 

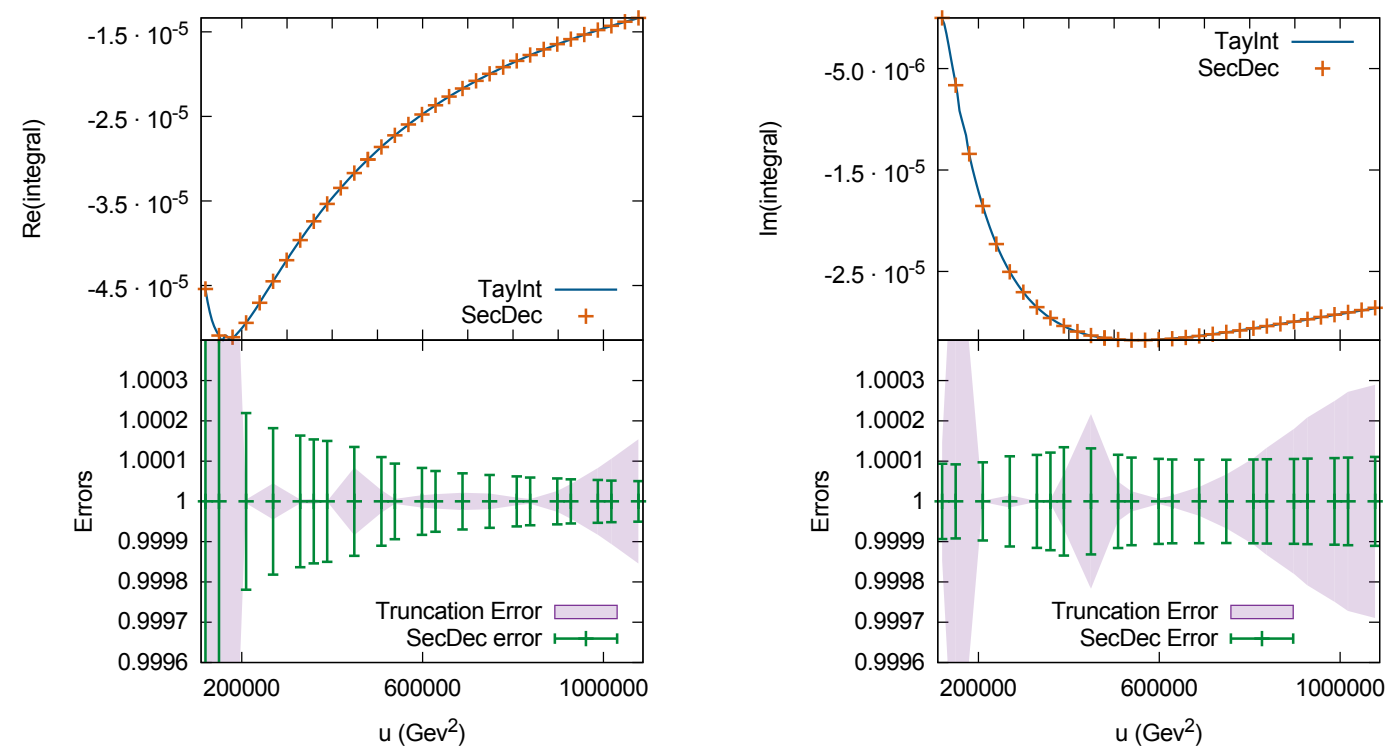

Figure 14. I10 over the threshold, calculated at $\mathcal{O}\left(\epsilon^{0}\right)$ with a sixth-order series expansion choosing $m_{2}=\frac{1}{\sqrt{2}} m_{1}$ and $m_{1}=173 \mathrm{GeV}$. The lower plots show the relative uncertainties of the TAYINT and numerical SECDEC results, respectively.

and the integrator VEGAS were chosen. Figure 15 is a zoom into the region of larger $u$ values. The TAYInT truncation errors in the lower half of the plot are shown in yellow, using a four-fold partitioning, and in lilac, using an eight-fold partitioning. A strong increase in accuracy can be observed when the integrand is partitioned more often. More specifically, the relative truncation errors decrease from $\mathcal{O}\left(10^{-4}\right)$ with 4 partitions, to $\mathcal{O}\left(10^{-5}\right)$ when 8 partitions are used.

A more thorough quantitative analysis of the impact of the partitioning is given in table 3, where the relative truncation error for the integral I10 is shown for different expansion orders and integral partitions. On the one hand, it shows that the accuracy increase by doubling the number of partitions is roughly equivalent to raising the order of the expansion by two. On the other hand, each doubling of the number of partitions leads to an order of magnitude gain in precision.

Close to thresholds, there can be occasional rapid changes at the endpoints of integration regions which lead to larger truncation errors, for example increases of $10 \%$ are observed for the $\mathcal{O}\left(\epsilon^{0}\right)$ coefficient of I10. Beyond $u=30 m_{1}^{2}$ in the kinematic region over the threshold, the points of rapid fluctuation move closer to the boundary of the integration region, however do not enter it. This also leads to a small reduction in the precision of the TAYINT results, however this loss is at the $0.01 \%$ level for the $\mathcal{O}\left(\epsilon^{0}\right)$ coefficient of I10. This is illustrated in figure 16 by plotting the first I10 sector near to the threshold, reasonably over the threshold, and very far over the threshold in $u$.

In figure 17 the fourth-order (with eight partitions) TAYINT $\mathcal{O}\left(\epsilon^{1}\right)$ result for the integral I10 is compared to the SECDEC result in the over threshold region, showing no drop 

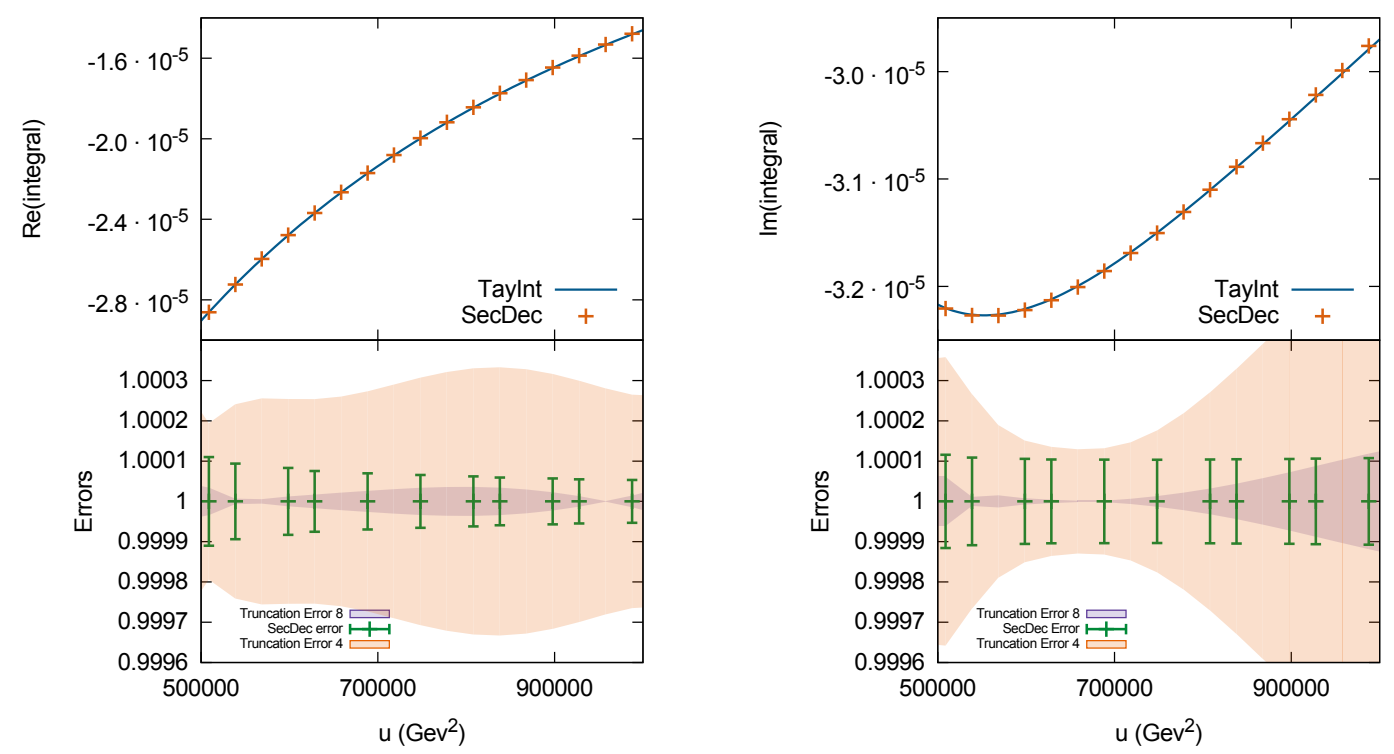

Figure 15. I10 calculated at $\mathcal{O}\left(\epsilon^{0}\right)$ with a sixth-order series expansion. The scale $u$ is over the $4 m_{1}^{2}$ threshold, with the near threshold region excluded, and $m_{2}=\frac{1}{\sqrt{2}} m_{1}, m_{1}=173 \mathrm{GeV}$. The lower plots show the relative TAYInT, with 4 and 8 partitions, and SECDEC uncertainties, respectively.

\begin{tabular}{|c|c|c|c|c|}
\hline \multicolumn{5}{|c|}{ Mean relative TAYINT truncation error } \\
\hline $\begin{array}{c}\text { Number of } \\
\text { Partitions }\end{array}$ & \multicolumn{2}{|c|}{8} \\
\hline Order & $\operatorname{Re}(\mathrm{I} 10)$ & $\operatorname{Im}(\mathrm{I} 10)$ & $\operatorname{Re}(\mathrm{I} 10)$ & $\operatorname{Im}(\mathrm{I} 10)$ \\
\hline 0 & 0.530165 & 0.623989 & 0.0812167 & 0.242449 \\
\hline 2 & 0.0221554 & 0.0242271 & 0.000642405 & 0.00237282 \\
\hline 4 & 0.00278254 & 0.00242541 & 0.000163342 & 0.000079292 \\
\hline 6 & 0.000284179 & 0.000281809 & 0.0000239721 & 0.000038864 \\
\hline
\end{tabular}

Table 3. The impact on the mean relative TAYInT truncation error of changing the order of the Taylor expansion and the number of partitions in the TAYINT algorithm applied to I10 at $\mathcal{O}\left(\epsilon^{0}\right)$. The kinematic region over which the mean is taken is given by $u \in\left[16 m_{1}^{2}, 32 m_{1}^{2}\right]=$ $[467864,957728] \mathrm{GeV}^{2}, m_{2}=\frac{1}{\sqrt{2}} m_{1}$ and $m_{1}=173 \mathrm{GeV}$.

in accuracy with respect to the lower order in $\epsilon$. The SECDEC results were computed using a relative accuracy of $10^{-3}$ with default numerical integration parameters and the integrator VEGAS.

In figure 18, the $\mathcal{O}\left(\epsilon^{2}\right)$ result for the integral I10 is compared to the SECDEC result in the over threshold region, again without diminished accuracy with respect to the lower orders in $\epsilon$. The results shown are based on a fourth-order Taylor expansion with sixteen partitions. A relative accuracy of $10^{-4}$ was used for the production of the SECDEC results. The $\mathcal{O}\left(\epsilon^{2}\right)$ result for I10 is obtained without OT3-4, as the $\mathcal{O}\left(\epsilon^{2}\right)$ coefficients of the subsector integrands cannot be integrated exactly. However, the same level of accuracy with respect 


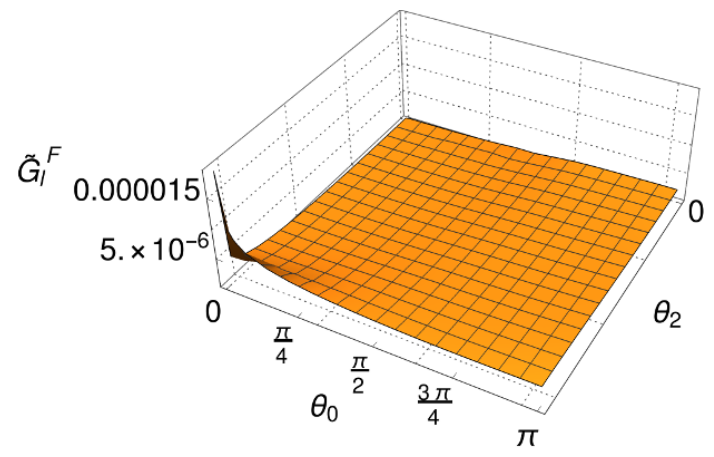

(a)

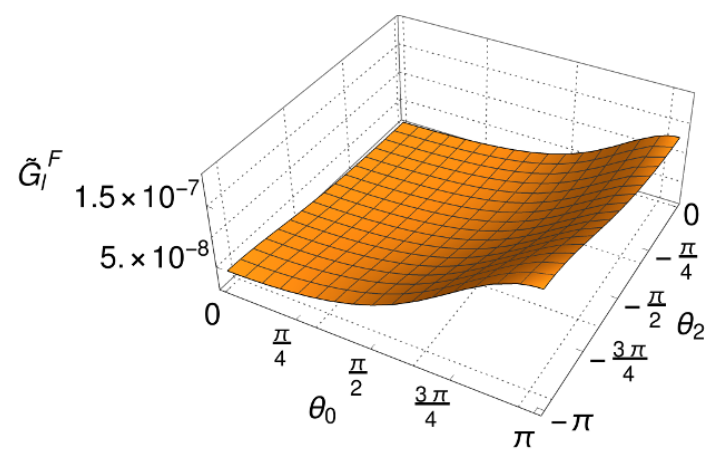

(b)

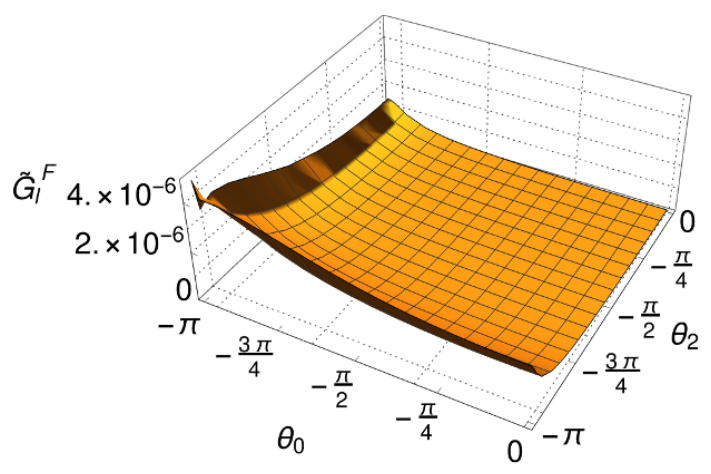

(c)

Figure 16. The first subsector of I10 at $\mathcal{O}\left(\epsilon^{0}\right)$ after OT3 at; (a) a near threshold point $u=$ $179574 \mathrm{GeV}^{2}$, (b) a point a reasonable distance over the threshold $u=748225 \mathrm{GeV}^{2}$, (c) a point very far over the threshold, $u=1017586 \mathrm{GeV}^{2}$. In all cases, $m_{2}=\frac{1}{\sqrt{2}} m_{1}$ and $m_{1}=173 \mathrm{GeV}$.

to the lower orders in $\epsilon$ is achieved by increasing the number of partitions used in OT5, with respect to the lower $\epsilon$ orders. To make the behaviour of the uncertainty estimates in the near threshold region clearer, more points are plotted in the $u \in\left[4 m_{1}^{2}, 6 m_{1}^{2}\right]$ region. It is important to observe that the $\mathcal{O}\left(\epsilon^{0}\right)$ and $\mathcal{O}\left(\epsilon^{2}\right)$ results for I10 of figure 14 and 18, each display a region (well above the threshold) with an apparent deterioration of the TAYINT error. This is not a result of the Taylor expansion having a smaller radius of convergence, as is the case near or well above the threshold. Rather, this is a parametric effect that arises from two of the subsector integrands exhibiting oscillatory behaviour around zero in the vicinity of these kinematical points. These result in enhanced numerical cancellations among consecutive orders of the expansion. As a consequence, the uncertainty is grossly overestimated by the truncation error.

In figure 19 the TAYINT approach is applied to the $\mathcal{O}\left(\epsilon^{0}\right)$ coefficient of the integral I39, with more propagators and scales, without a loss in accuracy compared to the simpler examples discussed above. For the calculation an eight-fold partitioning and a fourth-order Taylor expansion was used. The TAYInT results agree well with the SECDEC results, and are stable over the whole kinematic region, given the truncation error only ever varies by $0.01 \%$. For the SECDEC results a relative accuracy of $10^{-3}$ was chosen. In figure 20, the 

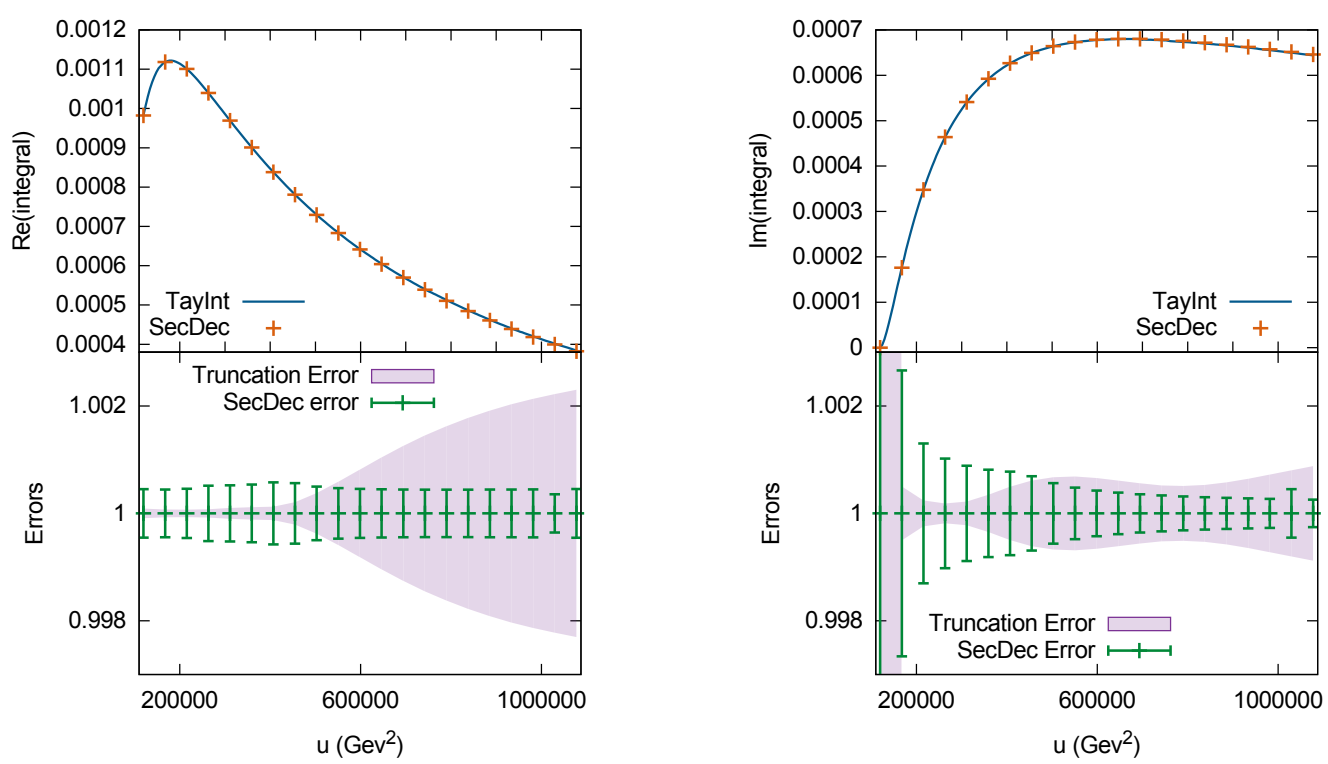

Figure 17. The I10 Integral calculated at $\mathcal{O}\left(\epsilon^{1}\right)$ with a fourth-order series expansion. The scale $u$ is over the $4 m_{1}^{2}$ threshold, with $m_{2}=\frac{1}{\sqrt{2}} m_{1}$ and $m_{1}=173 \mathrm{GeV}$. The lower plots show the relative uncertainties of the TAYINT and numerical SECDEC results, respectively.
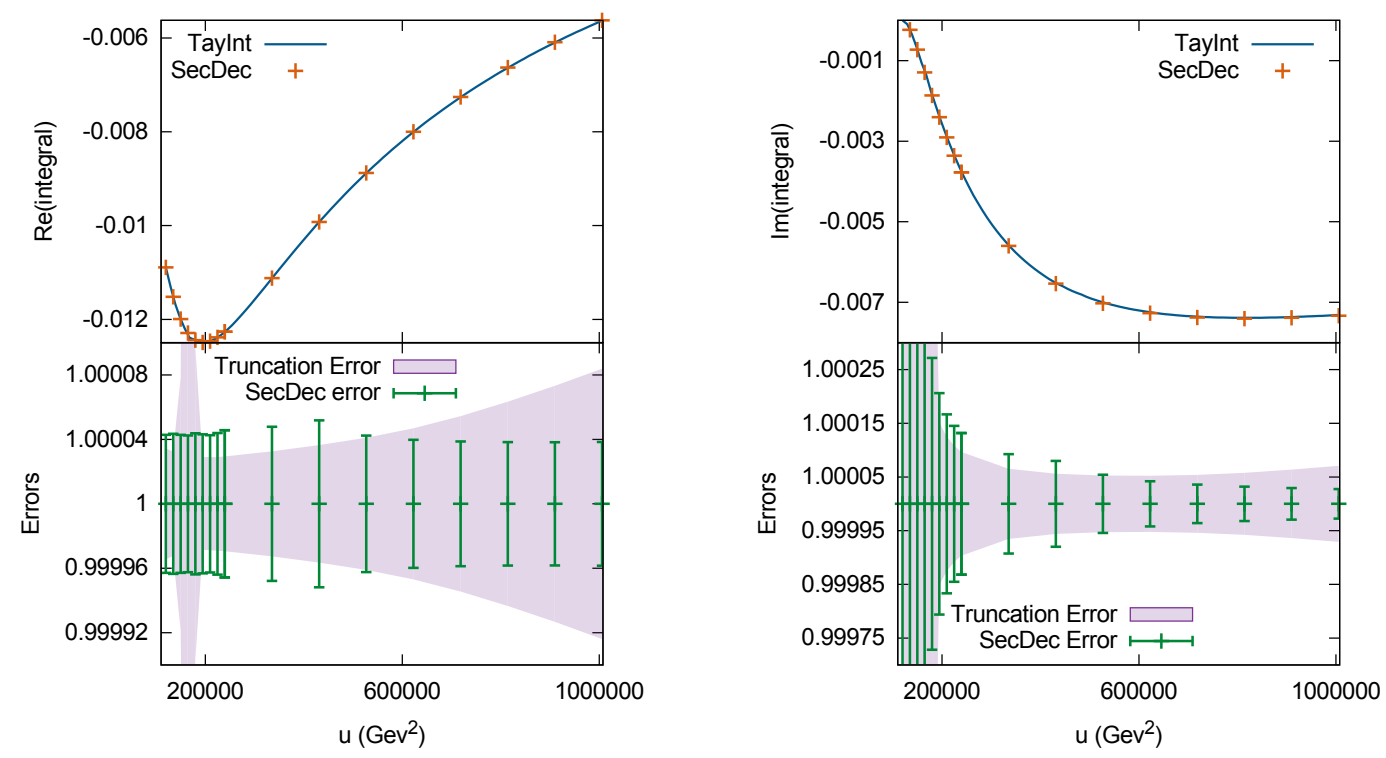

Figure 18. The I10 Integral calculated at $\mathcal{O}\left(\epsilon^{2}\right)$ with a fourth-order series expansion. The scale $u$ is over the $4 m_{1}^{2}$ threshold, with $m_{2}=\frac{1}{\sqrt{2}} m_{1}$ and $m_{1}=173 \mathrm{GeV}$. The lower plots show the relative TAYINT and SECDEC errors, respectively.

TAYInT approach is applied to the $\mathcal{O}\left(\epsilon^{1}\right)$ coefficient of the integral I39. No exact integration could be performed and a fourth-order Taylor expansion with six partitions was used, and the TAYINT results are consistent with the SECDEC results across their full kinematic range. 

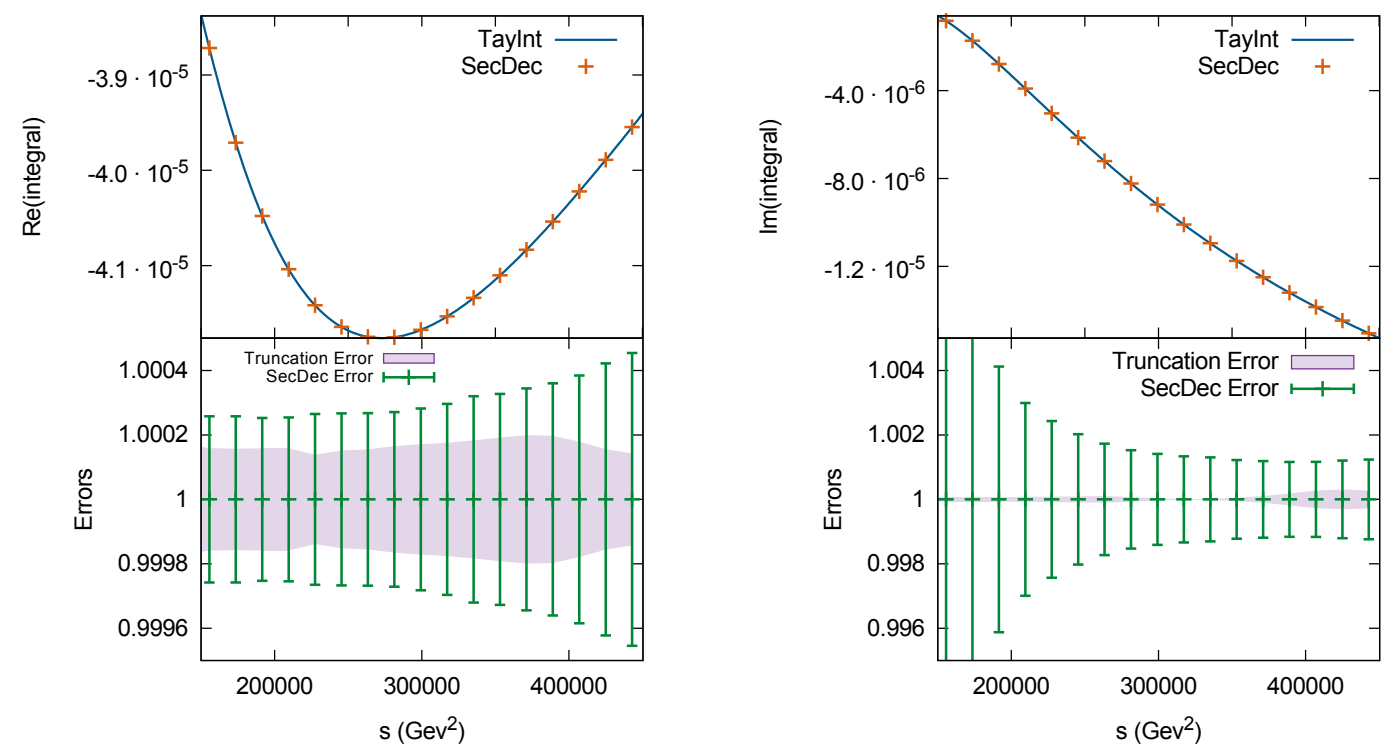

Figure 19. The I39 Integral calculated at $\mathcal{O}\left(\epsilon^{0}\right)$ with a fourth-order series expansion. The scale $s$ is over the $4 m_{1}^{2}$ threshold, with $u=-59858 \mathrm{GeV}^{2}, m_{2}=\frac{1}{\sqrt{2}} m_{1}$ and $m_{1}=173 \mathrm{GeV}$. The lower plots show the relative TAYINT and SECDEC uncertainties, respectively.

Given the complexity of the integrand, a comparably small number of partitions was used, however no further precision was needed to obtain agreement between the SECDEC and TAYINT result within the associated uncertainty. To put this into context, the TAYINT result, $0.000934066+0.000126179 \mathrm{i}$, at the ninth kinematic point at which SECDEC is evaluated, the result of which is $0.000933183+0.000127422 \mathrm{i}$, has an associated absolute uncertainty of $3.5495 \cdot 10^{-6}+3.48459 \cdot 10^{-6} \mathrm{i}$. Thus there is agreement between TAYINT and SecDec within the TAYInt uncertainty. For the sake of comparison, the SecDeC results for figure 20 were obtained asking for a relative accuracy of $10^{-3}$. In figure 21 , the $\mathcal{O}\left(\epsilon^{2}\right)$ coefficient of the integral I39 is calculated with TAYINT. No exact integration could be performed and a fourth-order Taylor expansion with eight partitions was used, and the algebraic TAYINT result coincides with the SECDEC results at the set of points considered above the $4 m_{1}^{2}$ threshold. The $\mathcal{O}\left(\epsilon^{2}\right)$ SECDEC results were obtained at a requested relative accuracy of $10^{-3}$. It is already apparent from figure 13 that the accuracy of the results obtained for I39, an integral for which there is, thus far, no analytic result, below $s=4 m_{1}^{2}$ is independent of the order in $\epsilon$. This is now shown to also be true over the $4 m_{1}^{2}$ threshold. By its application to integrals with increasing numbers of scales and propagators, for different kinematic hierarchies and to order $\mathcal{O}\left(\epsilon^{2}\right)$, the versatility of TAYINT has been demonstrated.

To reinforce this, the mean difference between the TAYINT and SECDEC results for I10, I21 and I39 over the threshold,

$$
\Delta=\frac{\sum_{i=1}^{\xi}\left(T_{l}^{F}\left(\left\{q_{i}\right\},\left\{m_{1}\right\}\right)-G_{\mathrm{SECDEC}}^{F}\left(\left\{q_{i}\right\},\left\{m_{1}\right\}\right)\right)}{\xi\left(G_{\mathrm{SECDEC}}^{F}\left(\left\{q_{i}\right\},\left\{m_{1}\right\}\right)\right)}
$$

is tabulated in table 4. $G_{\text {SECDEC }}^{F}(\{q\},\{m\})$ is the SECDEC result for the full Feynman 

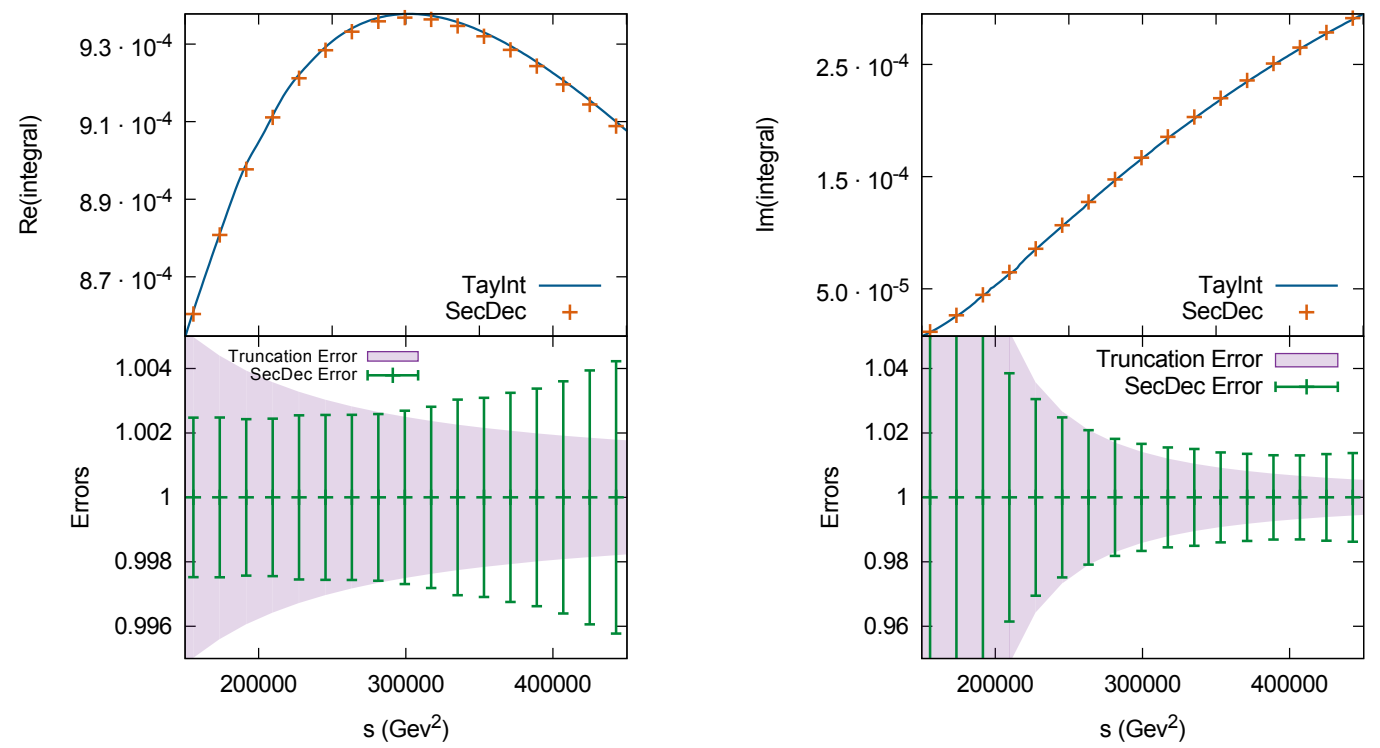

Figure 20. The I39 Integral calculated at $\mathcal{O}\left(\epsilon^{1}\right)$ with a fourth-order series expansion. The scale $s$ is over the $4 m_{1}^{2}$ threshold, with $u=-59858 \mathrm{GeV}^{2}, m_{2}=\frac{1}{\sqrt{2}} m_{1}$ and $m_{1}=173 \mathrm{GeV}$. The lower plots show the relative TAYINT and SECDEC uncertainties, respectively.
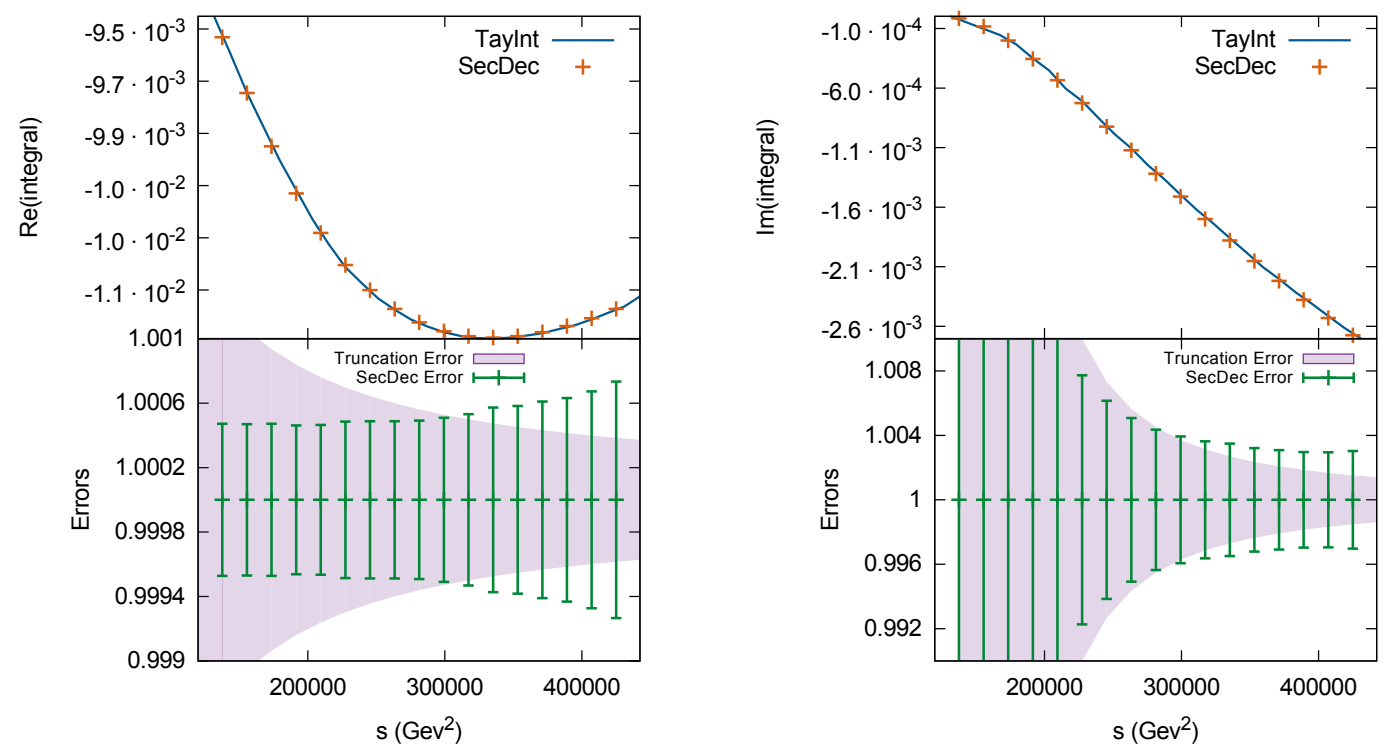

Figure 21. The I39 Integral calculated at $\mathcal{O}\left(\epsilon^{2}\right)$ with a fourth-order series expansion. The scale $s$ is over the $4 m_{1}^{2}$ threshold, with $u=-59858 \mathrm{GeV}^{2}, m_{2}=\frac{1}{\sqrt{2}} m_{1}$ and $m_{1}=173 \mathrm{GeV}$. The lower plots show the relative TAYINT and SECDEC uncertainties, respectively.

integral $G^{F}(\{q\},\{m\})$. The index $i$ runs over the $\xi$ different kinematic points at which results for each integral were generated, so $\left\{q_{\xi}\right\}=\left\{16 m_{1}^{2},-2 m_{1}^{2}, 0.5 m_{1}^{2}\right\}$ for I39. In all cases $\{m\}=\left\{m_{1}\right\}$. All TAYINT results are based on a sixth-order, four partition, series 


\begin{tabular}{|l|l|l|}
\hline Graph & $\operatorname{Re}(\Delta)$ & $\operatorname{Im}(\Delta)$ \\
\hline I10 & 0.000658179 & 0.000270775 \\
\hline I21 & 0.00126601 & 0.000277579 \\
\hline I39 & 0.0000763027 & 0.0000668706 \\
\hline
\end{tabular}

Table 4. The mean difference $\Delta$ between TAYInT and SECDEC, normalised to the SECDEC result. The kinematic points are $u \in\left[4 m_{1}^{2}, 36 m_{1}^{2}\right]=[119716,1077444] \mathrm{GeV}^{2}$ for I10 and I21 and $s \in\left[4 m_{1}^{2}, 16 m_{1}^{2}\right]=[119716,478864] \mathrm{GeV}^{2}$ for I39.
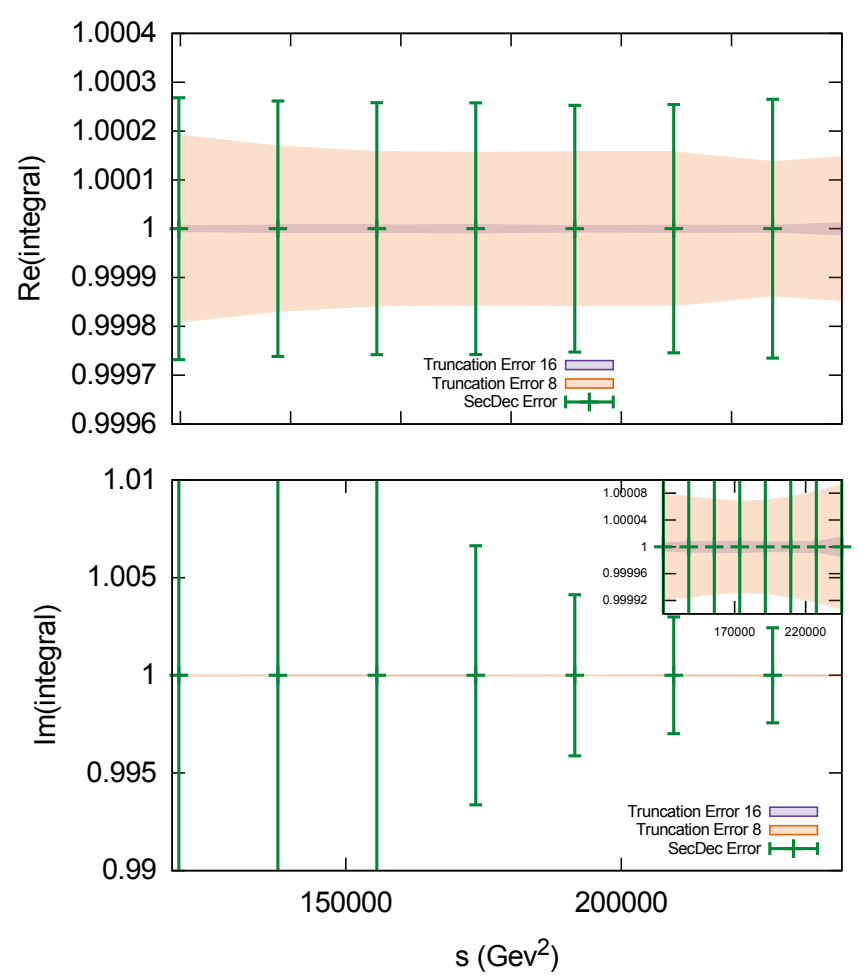

Figure 22. The relative TAYINT uncertainty obtained with 8 and 16 partitions respectively, for the integral I39 at $\mathcal{O}\left(\epsilon^{0}\right)$ above the threshold, with $u=-59858 \mathrm{GeV}^{2}, m_{2}=\frac{1}{\sqrt{2}} m_{1}$ and $m_{1}=173 \mathrm{GeV}$.

expansion. The results for I10 are $792.8 \mathrm{MB}$ in size, while for I39 they total $1.3488 \mathrm{~GB}$. The file sizes refer to their unsimplified version. Using the plain Simplify command in MATHEMATICA the size of the results can already be reduced by a factor of 1.33. Furthermore, removal of floating point zeros, 0 ., that appear in the results files leads to a reduction in size by a factor of 2.6 from the sizes quoted here. With the planned improved automation of TAYINT, the simplification of the result files will also be addressed. One of the most attractive features is that the precision of the TAYINT results is independent of the $\epsilon$ order. Nevertheless the computation time required, both for the configuration determination and the actual calculation, increases significantly when going to higher orders in $\epsilon$.

In figure 22 the truncation errors obtained using 8 and 16 partitions are plotted for the integral I39 at $\mathcal{O}\left(\epsilon^{0}\right)$ in yellow and purple bands, respectively. The $y$-axis is truncated 
so that the larger ratios near the threshold, due to the TAYINT and SECDEC results being consistent with zero, cannot be seen. However, given the size of the SECDEC errors in the imaginary part, an inset provides a closer look at the TAYINT error bands. The TAYINT result is based on a fourth-order series expansion. The SECDEC results have a relative accuracy of $10^{-3}$. The TAYINT uncertainties decrease by an order of magnitude when the number of partitions is doubled, replicating the effect seen for the I10 integral. The average SECDEC evaluation time at a phase-space point increases by a factor of 1.6 for each order of magnitude increase in relative precision, while, due to the fact that the TAYINT algorithm produces an algebraic integral library, analytic in the kinematical scales, the evaluation using the TAYINT result is always instantaneous.

\section{Conclusion}

TAYINT is a new algorithm which calculates systematic approximations to Feynman integrals algebraically in the kinematic invariants such that the results have validity in all kinematic regions and can be made arbitrarily precise.

The algorithm takes the propagators as input and works with subsector integrals generated by the program SECDEC. The actual integration is facilitated via a Taylor expansion in the integration parameters. The accuracy is bolstered by conformal mappings and partitioning of the integrand before performing the Taylor expansion. The validity over threshold is ensured by performing a variable transformation which implements the correct analytical continuation of the integrand into the complex plane. Results can be obtained to higher orders in the dimensional regulator $\epsilon$, both above and below mass thresholds.

We demonstrated the application of TAYINT on two-loop three-point and four-point integrals with an internal mass, and used these examples to illustrate several features and virtues of the TAYINT algorithm.

The TAYInT algorithm expands upon the SECDEC framework, and is in principle applicable to Feynman integrals with any number of loops and any number of kinematical scales. Its practical application could be limited by the algebraic complexity of intermediate expressions and final expansions, or the availability of a quasi-finite basis. Based on the proof-of-principle applications considered here, we are confident that our expansion algorithm can be fully automated and applied to many two-loop and three-loop problems of high phenomenological interest, where closed analytical expressions can not be obtained.

\section{Acknowledgments}

We thank Erik Panzer, Andreas von Manteuffel and Dominik Kara for insightful discussions. This research was supported in part by the Swiss National Science Foundation (SNF) under contract 200020-175595, by the Research Executive Agency (REA) of the European Union through the ERC Grants MC@NNLO (340983) and MathAm (395568) and the National Science Foundation under Grant No. NSF PHY11-25915. 
Open Access. This article is distributed under the terms of the Creative Commons Attribution License (CC-BY 4.0), which permits any use, distribution and reproduction in any medium, provided the original author(s) and source are credited.

\section{References}

[1] S. Weinberg, The quantum theory of fields, volume 1, Cambridge University Press, Cambridge U.K. (2008), pg. 497.

[2] A.V. Kotikov, Differential equations method: New technique for massive Feynman diagrams calculation, Phys. Lett. B 254 (1991) 158 [INSPIRE].

[3] E. Remiddi, Differential equations for Feynman graph amplitudes, Nuovo Cim. A 110 (1997) 1435 [hep-th/9711188] [INSPIRE].

[4] M. Caffo, H. Czyz, S. Laporta and E. Remiddi, Master equations for master amplitudes, Acta Phys. Polon. B 29 (1998) 2627 [hep-th/9807119] [INSPIRE].

[5] M. Caffo, H. Czyz, S. Laporta and E. Remiddi, The Master differential equations for the two loop sunrise selfmass amplitudes, Nuovo Cim. A 111 (1998) 365 [hep-th/9805118] [INSPIRE].

[6] T. Gehrmann and E. Remiddi, Differential equations for two loop four point functions, Nucl. Phys. B 580 (2000) 485 [hep-ph/9912329] [INSPIRE].

[7] J.M. Henn, Multiloop integrals in dimensional regularization made simple, Phys. Rev. Lett. 110 (2013) 251601 [arXiv:1304.1806] [INSPIRE].

[8] R.N. Lee, A.V. Smirnov and V.A. Smirnov, Solving differential equations for Feynman integrals by expansions near singular points, JHEP 03 (2018) 008 [arXiv: 1709.07525] [INSPIRE].

[9] X. Liu, Y.-Q. Ma and C.-Y. Wang, A Systematic and Efficient Method to Compute Multi-loop Master Integrals, Phys. Lett. B 779 (2018) 353 [arXiv:1711.09572] [INSPIRE].

[10] H. Poincaré, Sur les groupes des équations lineéaires, Acta Math. 4 (1883) 215.

[11] E. Kummer, Über die Transzendenten, welche aus wiederholten Integrationen rationaler Formeln entstehen, J. Reine Angew. Math. 21 (1840) 74.

[12] N. Nielsen, Der eulersche dilogarithmus und seine verallgemeinerungen, Nova Acta Leopoldina (Halle) $90 \mathbf{1 2 3}$ (1909).

[13] A.B. Goncharov, Geometry of configurations, polylogarithms, and motivic cohomology, Adv. Math. 114 (1995) 197.

[14] A.B. Goncharov, Multiple polylogarithms, cyclotomy and modular complexes, Math. Res. Lett. 5 (1998) 497 [arXiv: 1105.2076] [INSPIRE].

[15] E. Remiddi and J.A.M. Vermaseren, Harmonic polylogarithms, Int. J. Mod. Phys. A 15 (2000) 725 [hep-ph/9905237] [INSPIRE].

[16] J. Vollinga and S. Weinzierl, Numerical evaluation of multiple polylogarithms, Comput. Phys. Commun. 167 (2005) 177 [hep-ph/0410259] [INSPIRE].

[17] A.B. Goncharov, M. Spradlin, C. Vergu and A. Volovich, Classical Polylogarithms for Amplitudes and Wilson Loops, Phys. Rev. Lett. 105 (2010) 151605 [arXiv:1006.5703] [INSPIRE]. 
[18] J. Ablinger, J. Blumlein and C. Schneider, Harmonic Sums and Polylogarithms Generated by Cyclotomic Polynomials, J. Math. Phys. 52 (2011) 102301 [arXiv:1105.6063] [InSPIRE].

[19] C. Duhr, Hopf algebras, coproducts and symbols: an application to Higgs boson amplitudes, JHEP 08 (2012) 043 [arXiv: 1203.0454] [INSPIRE].

[20] T. Gehrmann and E. Remiddi, Two loop master integrals for $\gamma^{*} \rightarrow 3$ jets: The Nonplanar topologies, Nucl. Phys. B 601 (2001) 287 [hep-ph/0101124] [INSPIRE].

[21] T. Gehrmann and E. Remiddi, Two loop master integrals for $\gamma^{*} \rightarrow 3$ jets: The Planar topologies, Nucl. Phys. B 601 (2001) 248 [hep-ph/0008287] [INSPIRE].

[22] R. Bonciani, P. Mastrolia and E. Remiddi, Master integrals for the two loop QCD virtual corrections to the forward backward asymmetry, Nucl. Phys. B 690 (2004) 138 [hep-ph/0311145] [INSPIRE].

[23] C. Anastasiou, S. Beerli, S. Bucherer, A. Daleo and Z. Kunszt, Two-loop amplitudes and master integrals for the production of a Higgs boson via a massive quark and a scalar-quark loop, JHEP 01 (2007) 082 [hep-ph/0611236] [INSPIRE].

$[24]$ T. Gehrmann, L. Tancredi and E. Weihs, Two-loop master integrals for $q \bar{q} \rightarrow V V$ : the planar topologies, JHEP 08 (2013) 070 [arXiv:1306.6344] [INSPIRE].

[25] J.M. Henn, K. Melnikov and V.A. Smirnov, Two-loop planar master integrals for the production of off-shell vector bosons in hadron collisions, JHEP 05 (2014) 090 [arXiv: 1402.7078] [INSPIRE].

[26] F. Caola, J.M. Henn, K. Melnikov and V.A. Smirnov, Non-planar master integrals for the production of two off-shell vector bosons in collisions of massless partons, JHEP 09 (2014) 043 [arXiv: 1404.5590] [INSPIRE].

[27] T. Gehrmann, A. von Manteuffel, L. Tancredi and E. Weihs, The two-loop master integrals for $q \bar{q} \rightarrow V V$, JHEP 06 (2014) 032 [arXiv: 1404.4853] [INSPIRE].

[28] C.G. Papadopoulos, D. Tommasini and C. Wever, The Pentabox Master Integrals with the Simplified Differential Equations approach, JHEP 04 (2016) 078 [arXiv:1511.09404] [INSPIRE].

[29] T. Gehrmann, J.M. Henn and N.A. Lo Presti, Analytic form of the two-loop planar five-gluon all-plus-helicity amplitude in QCD, Phys. Rev. Lett. 116 (2016) 062001 [arXiv:1511.05409] [INSPIRE].

[30] J.M. Henn, A.V. Smirnov and V.A. Smirnov, Analytic results for planar three-loop integrals for massive form factors, JHEP 12 (2016) 144 [arXiv:1611.06523] [INSPIRE].

[31] R. Bonciani, V. Del Duca, H. Frellesvig, J.M. Henn, F. Moriello and V.A. Smirnov, Two-loop planar master integrals for Higgs $\rightarrow 3$ partons with full heavy-quark mass dependence, JHEP 12 (2016) 096 [arXiv: 1609.06685] [INSPIRE].

[32] M. Becchetti and R. Bonciani, Two-Loop Master Integrals for the Planar QCD Massive Corrections to Di-photon and Di-jet Hadro-production, JHEP 01 (2018) 048 [arXiv: 1712.02537] [INSPIRE].

[33] F.C.S. Brown and A. Levin, Multiple Elliptic Polylogarithms, arXiv:1110.6917.

[34] J. Broedel, C. Duhr, F. Dulat and L. Tancredi, Elliptic polylogarithms and iterated integrals on elliptic curves. Part I: general formalism, JHEP 05 (2018) 093 [arXiv: 1712.07089] [INSPIRE]. 
[35] J. Broedel, C. Duhr, F. Dulat and L. Tancredi, Elliptic polylogarithms and iterated integrals on elliptic curves II: an application to the sunrise integral, Phys. Rev. D 97 (2018) 116009 [arXiv: 1712.07095] [INSPIRE].

[36] E. Remiddi and L. Tancredi, An Elliptic Generalization of Multiple Polylogarithms, Nucl. Phys. B 925 (2017) 212 [arXiv: 1709.03622] [InSPIRE].

[37] A. von Manteuffel and L. Tancredi, A non-planar two-loop three-point function beyond multiple polylogarithms, JHEP 06 (2017) 127 [arXiv:1701.05905] [INSPIRE].

[38] L. Adams and S. Weinzierl, The $\varepsilon$-form of the differential equations for Feynman integrals in the elliptic case, Phys. Lett. B 781 (2018) 270 [arXiv:1802.05020] [INSPIRE].

[39] B. Mistlberger, Higgs boson production at hadron colliders at $N^{3} L O$ in $Q C D$, JHEP 05 (2018) 028 [arXiv : 1802.00833] [INSPIRE].

[40] K. Hepp, Proof of the bogoliubov-parasiuk theorem on renormalization, Commun. Math. Phys. 2 (1966) 301.

[41] M. Roth and A. Denner, High-energy approximation of one loop Feynman integrals, Nucl. Phys. B 479 (1996) 495 [hep-ph/9605420] [INSPIRE].

[42] T. Binoth and G. Heinrich, An automatized algorithm to compute infrared divergent multiloop integrals, Nucl. Phys. B 585 (2000) 741 [hep-ph/0004013] [INSPIRE].

[43] G. Heinrich, Sector Decomposition, Int. J. Mod. Phys. A 23 (2008) 1457 [arXiv:0803.4177] [INSPIRE].

[44] C. Bogner and S. Weinzierl, Resolution of singularities for multi-loop integrals, Comput. Phys. Commun. 178 (2008) 596 [arXiv:0709.4092] [inSPIRE].

[45] A.V. Smirnov and M.N. Tentyukov, Feynman Integral Evaluation by a Sector decomposiTion Approach (FIESTA), Comput. Phys. Commun. 180 (2009) 735 [arXiv:0807.4129] [INSPIRE].

[46] A.V. Smirnov, V.A. Smirnov and M. Tentyukov, FIESTA 2: Parallelizeable multiloop numerical calculations, Comput. Phys. Commun. 182 (2011) 790 [arXiv:0912.0158] [INSPIRE].

[47] A.V. Smirnov, FIESTA 3: cluster-parallelizable multiloop numerical calculations in physical regions, Comput. Phys. Commun. 185 (2014) 2090 [arXiv:1312.3186] [INSPIRE].

[48] J. Gluza, K. Kajda, T. Riemann and V. Yundin, Numerical Evaluation of Tensor Feynman Integrals in Euclidean Kinematics, Eur. Phys. J. C 71 (2011) 1516 [arXiv:1010.1667] [INSPIRE].

[49] J. Carter and G. Heinrich, SecDec: A general program for sector decomposition, Comput. Phys. Commun. 182 (2011) 1566 [arXiv:1011.5493] [INSPIRE].

[50] S. Borowka, J. Carter and G. Heinrich, Numerical Evaluation of Multi-Loop Integrals for Arbitrary Kinematics with SecDec 2.0, Comput. Phys. Commun. 184 (2013) 396 [arXiv: 1204.4152] [INSPIRE].

[51] S. Borowka, G. Heinrich, S.P. Jones, M. Kerner, J. Schlenk and T. Zirke, SecDec-3.0: numerical evaluation of multi-scale integrals beyond one loop, Comput. Phys. Commun. 196 (2015) 470 [arXiv: 1502.06595] [INSPIRE].

[52] S. Borowka et al., pySecDec: a toolbox for the numerical evaluation of multi-scale integrals, Comput. Phys. Commun. 222 (2018) 313 [arXiv:1703.09692] [INSPIRE]. 
[53] S. Borowka, T. Hahn, S. Heinemeyer, G. Heinrich and W. Hollik, Momentum-dependent two-loop QCD corrections to the neutral Higgs-boson masses in the MSSM, Eur. Phys. J. C 74 (2014) 2994 [arXiv: 1404.7074] [INSPIRE].

[54] S. Borowka et al., Higgs Boson Pair Production in Gluon Fusion at Next-to-Leading Order with Full Top-Quark Mass Dependence, Phys. Rev. Lett. 117 (2016) 012001 [arXiv: 1604.06447] [inSPIRE].

[55] S. Borowka et al., Full top quark mass dependence in Higgs boson pair production at NLO, JHEP 10 (2016) 107 [arXiv: 1608.04798] [INSPIRE].

[56] S.P. Jones, M. Kerner and G. Luisoni, Next-to-Leading-Order QCD Corrections to Higgs Boson Plus Jet Production with Full Top-Quark Mass Dependence, Phys. Rev. Lett. 120 (2018) 162001 [arXiv: 1802.00349] [INSPIRE].

[57] S. Borowka, S. Paßehr and G. Weiglein, Complete two-loop QCD contributions to the lightest Higgs-boson mass in the MSSM with complex parameters, Eur. Phys. J. C 78 (2018) 576 [arXiv: 1802.09886] [INSPIRE].

[58] E. Panzer, On hyperlogarithms and Feynman integrals with divergences and many scales, JHEP 03 (2014) 071 [arXiv: 1401.4361] [INSPIRE].

[59] A. von Manteuffel, E. Panzer and R.M. Schabinger, A quasi-finite basis for multi-loop Feynman integrals, JHEP 02 (2015) 120 [arXiv:1411.7392] [INSPIRE].

[60] A. von Manteuffel and C. Studerus, Reduze 2 - Distributed Feynman Integral Reduction, arXiv: 1201.4330 [INSPIRE].

[61] C.W. Bauer, A. Frink and R. Kreckel, Introduction to the GiNaC framework for symbolic computation within the C++ programming language, J. Symb. Comput. 33 (2000) 1 [cs/0004015] [INSPIRE].

[62] Mathematica, Copyright by Wolfram Research.

[63] J.A.M. Vermaseren, New features of FORM, math-ph/0010025 [INSPIRE].

[64] J. Kuipers, T. Ueda and J.A.M. Vermaseren, Code Optimization in FORM, Comput. Phys. Commun. 189 (2015) 1 [arXiv:1310.7007] [INSPIRE].

[65] F.V. Tkachov, A Theorem on Analytical Calculability of Four Loop Renormalization Group Functions, Phys. Lett. B 100 (1981) 65.

[66] K.G. Chetyrkin and F.V. Tkachov, Integration by Parts: The Algorithm to Calculate B-functions in 4 Loops, Nucl. Phys. B 192 (1981) 159 [INSPIRE].

[67] S. Laporta, High precision calculation of multiloop Feynman integrals by difference equations, Int. J. Mod. Phys. A 15 (2000) 5087 [hep-ph/0102033] [INSPIRE].

[68] T. Kaneko and T. Ueda, A Geometric method of sector decomposition, Comput. Phys. Commun. 181 (2010) 1352 [arXiv:0908. 2897] [INSPIRE].

[69] T. Kaneko and T. Ueda, Sector Decomposition Via Computational Geometry, PoS (ACAT2010) 082 [arXiv: 1004.5490] [INSPIRE].

[70] H. Cheng and T. Wu, Expanding Protons: Scattering at High Energies, MIT Press, Cambridge U.S.A. (1987).

[71] V.A. Smirnov, Feynman integral calculus, Springer, Berlin Germany (2006). 
[72] R. Bonciani, P. Mastrolia and E. Remiddi, Vertex diagrams for the QED form-factors at the two loop level, Nucl. Phys. B 661 (2003) 289 [Erratum ibid. B 702 (2004) 359] [hep-ph/0301170] [INSPIRE].

[73] T. Gehrmann, S. Guns and D. Kara, The rare decay $H \rightarrow Z \gamma$ in perturbative $Q C D$, JHEP 09 (2015) 038 [arXiv: 1505.00561] [INSPIRE].

[74] A. Primo and L. Tancredi, On the maximal cut of Feynman integrals and the solution of their differential equations, Nucl. Phys. B 916 (2017) 94 [arXiv: 1610.08397] [InSPIRE].

[75] K. Melnikov, L. Tancredi and C. Wever, Two-loop amplitudes for $q g \rightarrow H q$ and $q \bar{q} \rightarrow H g$ mediated by a nearly massless quark, Phys. Rev. D 95 (2017) 054012 [arXiv:1702.00426] [INSPIRE].

[76] G.P. Lepage, A New Algorithm for Adaptive Multidimensional Integration, J. Comput. Phys. 27 (1978) 192 [INSPIRE]. 